TRANSACTIONS OF THE

AMERICAN MATHEMATICAL SOCIETY

Volume 350, Number 3, March 1998, Pages 1079-1102

S $0002-9947(98) 02038-8$

\title{
GALOIS RIGIDITY OF PRO- $l$ PURE BRAID GROUPS OF ALGEBRAIC CURVES
}

\author{
HIROAKI NAKAMURA AND NAOTAKE TAKAO
}

\begin{abstract}
In this paper, Grothendieck's anabelian conjecture on the pro- $l$ fundamental groups of configuration spaces of hyperbolic curves is reduced to the conjecture on those of single hyperbolic curves. This is done by estimating effectively the Galois equivariant automorphism group of the pro- $l$ braid group on the curve. The process of the proof involves the complete determination of the groups of graded automorphisms of the graded Lie algebras associated to the weight filtration of the braid groups on Riemann surfaces.
\end{abstract}

\section{INTRODUCTION AND MAIN RESUlTS}

Let $X$ be an absolutely irreducible algebraic variety defined over a number field $k$. Then, as is well-known, the profinite fundamental group $\pi_{1}(X)$ is a group extension of the absolute Galois group $G_{k}$ of $k$ by the geometric fundamental group $\pi_{1}\left(X_{\bar{k}}\right)$. With this extension structure of $\pi_{1}(X)$, one can associate a canonical "exterior Galois representation" into the outer automorphism group $\pi_{1}\left(X_{\bar{k}}\right)$ :

$$
\varphi_{X}: G_{k} \rightarrow \operatorname{Out} \pi_{1}\left(X_{\bar{k}}\right) .
$$

If this Galois image is large and non-abelian enough, then the Galois centralizer, the centralizer of the Galois image in Out $\pi_{1}\left(X_{\bar{k}}\right)$, would reflect effectively the $k$ automorphism group of $X$. This is one of the most primitive forms of Grothendieck's anabelian Tate conjectures ([G1] [G2]) suggesting to control the geometry of a certain class of varieties by the arithmetic fundamental groups.

If a rational prime $l$ is fixed, then it is possible to modify the above conjecture by replacing $\pi_{1}\left(X_{\bar{k}}\right)$ by its maximal pro-l quotient $\pi_{1}\left(X_{\bar{k}}\right)(l)$. The purpose of this paper is to consider the pro- $l$ version of Grothendieck's problem for special types of varieties $X$, i.e., the configuration spaces of curves. To be more precise, let $C$ be a nonsingular (not necessarily complete) hyperbolic curve over $k$, and define the $r$-dimensional configuration space of $C$ by

$$
C^{(r)}=\left\{\left(x_{1}, \ldots, x_{r}\right) \in C^{r} \mid x_{i} \neq x_{j}(i \neq j)\right\} .
$$

Then the geometric fundamental group of $C^{(r)}$ is isomorphic to the profinite completion of the braid group with $r$-strings supported on the Riemann surface of the complex points of $C$. Let

$$
\varphi_{C^{(r)}}^{(l)}: G_{k} \rightarrow \text { Out } \pi_{1}\left(C_{\bar{k}}^{(r)}\right)(l)
$$

Received by the editors September 10, 1995.

1991 Mathematics Subject Classification. Primary 14E20; Secondary 20F34, 20 F36.

Key words and phrases. Galois representation, anabelian geometry, braid group.

(C)1998 American Mathematical Society 
be the associated pro- $l$ exterior Galois representation, and let $\operatorname{Out}_{G_{k}} \pi_{1}\left(C_{\bar{k}}^{(r)}\right)(l)$ denote the centralizer of the Galois image of $\varphi_{C^{(r)}}^{(l)}$. We have then a canonical homomorphism

$$
\Phi_{C^{(r)}}^{(l)}: \operatorname{Aut}_{k} C^{(r)} \rightarrow \operatorname{Out}_{G_{k}} \pi_{1}\left(C_{\bar{k}}^{(r)}\right)(l)
$$

This must be an injective mapping for purely geometric reasons, though we shall give a Galois theoretic proof of the injectivity later in the proof of (4.5). Our main result of this paper is the following.

Theorem A (4.5). Let $C$ be a nonsingular hyperbolic curve over a number field $k$ such that $C_{\bar{k}}$ is not isomorphic to $\mathbf{P}^{1}-\{0,1, \infty\}$. Then there exists a sequence of injective homomorphisms:

$$
\operatorname{Aut}_{k} C \times S_{r+\varepsilon} \hookrightarrow \operatorname{Aut}_{k} C^{(r)} \hookrightarrow \operatorname{Out}_{G_{k}} \pi_{1}\left(C_{\bar{k}}^{(r)}\right)(l) \hookrightarrow \operatorname{Out}_{G_{k}} \pi_{1}\left(C_{\bar{k}}\right)(l) \times S_{r+\varepsilon},
$$

where $S_{r+\varepsilon}$ is the symmetric group of degree $r+\varepsilon$, and $\varepsilon=1$ if $C$ is an elliptic curve minus one $k$-rational point, and 0 otherwise.

The above theorem expands a previous result for $C=\mathbf{P}^{1}-\{0,1, \infty\}$ asserting that $\mathrm{Out}_{G_{k}} \pi_{1}\left(C^{(r)}\right)(l)$ is isomorphic to $\operatorname{Aut}_{k} C^{(r)}\left(\cong S_{r+3}\right)([\mathrm{N} 1]-[\mathrm{N} 2])$. From Theorem A above, we immediately obtain the following fact.

Corollary B. Let $C / k$ be as in Theorem A. If $\operatorname{Out}_{G_{k}} \pi_{1}\left(C_{\bar{k}}\right)(l)$ is a finite group, then $\operatorname{Out}_{G_{k}} \pi_{1}\left(C_{\bar{k}}^{(r)}\right)(l)(r=1,2, \ldots)$ are also finite groups. And if

$$
\operatorname{Aut}_{k}(C) \cong \operatorname{Out}_{G_{k}} \pi_{1}\left(C_{\bar{k}}\right)(l),
$$

then also

$$
\operatorname{Aut}_{k}\left(C^{(r)}\right) \cong \operatorname{Out}_{G_{k}} \pi_{1}\left(C_{\bar{k}}^{(r)}\right)(l) \quad(r=1,2, \ldots)
$$

This result supports the following pro-l "anabelian" Tate conjecture on single hyperbolic curves to higher dimensional configuration varieties.

Conjecture $\mathbf{C}$ (cf. [N2], p.75). Let $C$ be a nonsingular hyperbolic curve over a number field $k$, and let $l$ be a prime number. Then

$$
\operatorname{Aut}_{k}(C) \cong \operatorname{Out}_{G_{k}} \pi_{1}\left(C_{\bar{k}}\right)(l) .
$$

In particular, Out $_{G_{k}} \pi_{1}\left(C_{\bar{k}}\right)(l)$ should be finite.

Note that there have been (infinitely) many curves $C / k$ shown to support the above conjecture (cf. $[\mathrm{NT}],[\mathrm{N} 4]-[\mathrm{N} 5],[\mathrm{Tk}]$ ). Our main ingredient of the present paper shows that the $l$-adic Lie technique used in [N2], 44 (inspired from Deligne [De]) can also be applied effectively to the pro- $l$ fundamental groups of the configuration spaces of curves. This approach is simpler than the direct approach developed in [N2], $\S 3$, although the latter one remains effective for more general pro- $\mathfrak{C}$ fundamental groups with $\mathfrak{C}$ being an "admissible" full class of finite groups (see loc. cit. for more details).

Our result might also be compared with a topological result by N.V. Ivanov [Iv] asserting that every automorphism of a discrete braid group of a Riemann surface must come from an inner automorphism of the mapping class group canonically containing the braid group in question. Our approach taken here is, however, of a rather algebraic nature and differs from Ivanov's topological approach. In effect, we establish a direct estimate of the automorphism group of the graded Lie algebra 
$\mathrm{Gr}_{K} \pi_{g, n}^{(r)}$ (over a field $K$ of characteristic 0 ) associated with the weight filtration of the braid group on an $n$-point punctured Riemann surface of genus $g$ with $r$ strings:

Theorem D (3.1). Suppose $2-2 g-n<0$ and $r \geq 2$. Then,

$$
\operatorname{Aut}\left(\operatorname{Gr}_{K} \pi_{g, n}^{(r)}\right) \cong \operatorname{GSp}(2 g, K) \times S_{n-\varepsilon} \times S_{r+\varepsilon},
$$

where

$$
\varepsilon:= \begin{cases}3 & \text { if }(g, n)=(0,3) \\ 1 & \text { if }(g, n)=(1,1) \\ 0 \quad \text { otherwise }\end{cases}
$$

and $\operatorname{GSp}(2 g, K)$ is the group of symplectic similitudes of degree $2 g(\operatorname{GSp}(0, K):=$ $\left.K^{\times}, S_{0}:=\{1\}\right)$.

This generalizes Lemma (4.1.3) of [N2] (originated from P. Deligne's suggestion). The method of the present paper is also fairly influenced by our experience of the previous joint work with R. Ueno [NTU] approaching Oda's problem by means of techniques of Ihara and Kaneko [Ih], [IhK]. (See also Matsumoto [Ma]).

The organization of this paper is as follows. In $\S 2$, we relate the Malcev completion and the pro- $l$ completion of a braid group, and introduce classes of (outer) automorphisms of pro- $l$ braid groups. The weight filtrations are introduced also for the Malcev braid groups. In $\S 3$, we compute the automorphism group of the graded Lie algebra associated with the weight filtration of the braid group, and prove Theorem D. The proof of Theorem A will be given in $\S 4$.

Note added. After our submitting the present paper, Conjecture $\mathrm{C}$ was proved affirmatively (in a stronger form) by S. Mochizuki [M2]. By virtue of his crucial result, our reduction argument of the present paper also terminates in its ideal form; i.e., all the inclusion relations in Theorem A turn out to be equalities, and the last isomorphy of Corollary B holds true for all $r$. See also Kawahara-Terasoma [KT], Mochizuki [M1], Pop [P], Tamagawa [Tm] for recent related progresses.

\section{Weight Filtration, MalceV COMPletion}

\section{Pro- $l$ completion and Malcev completion.}

(2.1) We shall begin by recalling basic facts about Malcev completions of nilpotent groups. Let $\Gamma$ be a finitely generated (discrete) nilpotent group. Consider the group algebra $\mathbb{Q}[\Gamma]$ and its completion with respect to the augmentation ideal $I \subset \mathbb{Q}[\Gamma]:$

$$
\hat{\mathbb{Q}}[\Gamma]=\lim _{r} \mathbb{Q}[\Gamma] / I^{r} .
$$

It is naturally a complete Hopf algebra equipped with the comultiplication $\Delta$ : $\hat{\mathbb{Q}}[\Gamma] \rightarrow \hat{\mathbb{Q}}[\Gamma] \hat{\otimes} \hat{\mathbb{Q}}[\Gamma]$ induced from $g \rightarrow g \hat{\otimes} g$ for $g \in \Gamma$. The Malcev completion $\mathcal{U} \Gamma$ (resp. the Malcev Lie algebra $\mathcal{L} \Gamma$ ) of $\Gamma$ is the subset of group-like elements (resp. Lie-like elements) defined by

$$
\begin{aligned}
\mathcal{U} \Gamma & =\{\gamma \in \hat{\mathbb{Q}}[\Gamma] \mid \Delta(\gamma)=\gamma \hat{\otimes} \gamma\} \\
\text { (resp. } \mathcal{L} \Gamma & =\{\lambda \in \hat{\mathbb{Q}}[\Gamma] \mid \Delta(\lambda)=\lambda \hat{\otimes} 1+1 \hat{\otimes} \lambda\}) .
\end{aligned}
$$

It follows that $\mathcal{U} \Gamma$ forms a group and $\mathcal{L} \Gamma$ forms a Lie algebra. The usual maps exp and $\log$ give bijections between $\mathcal{U} \Gamma$ and $\mathcal{L} \Gamma$. The pair of the group $\mathcal{U} \Gamma$ and 
the natural map $j: \Gamma \rightarrow \mathcal{U} \Gamma$ is known to be characterized for $\Gamma$ by the following properties (cf. [Q], Appendix A):

(1) $\mathcal{U} \Gamma$ is a uniquely divisible group;

(2) The kernel of $j$ is the maximal torsion subgroup of $\Gamma$;

(3) For each $\gamma \in \mathcal{U} \Gamma$ there exists $n \geq 1$ with $\gamma^{n} \in j(\Gamma)$.

(2.2) As is well-known, the torsion elements of $\Gamma$ form a subgroup $T$. The quotient group $\Gamma / T$ is then torsion-free, and it is known that $\Gamma / T$ can be realized as a group of upper unitriangular matrices of $\mathrm{GL}_{n}(\mathbb{Q})$ for some $n>0$ (e.g. $[\mathrm{W}], 11.4$ ). The image of $\Gamma / T$ by the logarithm of unitriangular matrices generates a vector subspace $W:=\mathbb{Q} \log (\Gamma / T)$ of the upper triangular matrices, and $\bar{\Gamma}:=\exp (W)$ forms a uniquely divisible unitriangular matrix group containing $\Gamma / T$. The natural mapping $j: \Gamma \rightarrow \Gamma / T \hookrightarrow \bar{\Gamma}$ satisfies the conditions (1)-(3) of (2.1); hence $\bar{\Gamma}$ can be regarded as the Malcev completion $\mathcal{U} \Gamma$. Since $W$ can be defined by a finite number of linear forms, $\bar{\Gamma}$ is defined by a finite number of polynomials on the space of matrices. Thus there exists a linear algebraic group $U \Gamma$ over $\mathbb{Q}$ with $U \Gamma(\mathbb{Q}) \cong \mathcal{U} \Gamma$. The structure of $U \Gamma$ is determined by the Lie algebra structure on $W=\log (U \Gamma(\mathbb{Q}))$, which is isomorphic to $\mathcal{L} \Gamma$. Therefore $U \Gamma / \mathbb{Q}$ is independent of the choice of realizations of $\Gamma / T$ as upper unitriangular matrix groups. We call $U \Gamma$ the algebraic hull of $\Gamma$.

(2.3) Fix a rational prime $l$. We will denote the group of the $\mathbb{Q}_{l}$-rational points $U \Gamma\left(\mathbb{Q}_{l}\right)$ by $\mathcal{U}_{l} \Gamma$. It can be realized as a group of upper unitriangular matrices of $\mathrm{GL}_{n}\left(\mathbb{Q}_{l}\right)$. Since $\Gamma$ is assumed to be finitely generated, we may assume that the image of the canonical homomorphism $j^{\prime}: \Gamma \rightarrow \mathcal{U}_{l} \Gamma$ is contained in the group of upper unitriangular matrices with $\mathbb{Z}_{l}$-entries, which is a pro-l group. Thus we obtain a mapping of the pro-l completion of $\Gamma$ into $\mathcal{U}_{l} \Gamma$ :

$$
j_{l}: \hat{\Gamma}(l) \longrightarrow \mathcal{U}_{l} \Gamma \text {. }
$$

This mapping $j_{l}$ is the unique homomorphism extending $j^{\prime}$ continuously with respect to the natural topology. Moreover, $j_{l}$ is a local isomorphism, i.e., induces an isomorphism of the associated Lie algebra Lie $(\hat{\Gamma}(l))$ of the $l$-adic analytic group $\hat{\Gamma}(l)$ onto $\mathcal{L}_{l} \Gamma \stackrel{\text { def }}{=} \mathcal{L} \Gamma \otimes_{\mathbb{Q}} \mathbb{Q}_{l}$ :

$$
\operatorname{Lie}\left(j_{l}\right): \operatorname{Lie}(\hat{\Gamma}(l)) \stackrel{\sim}{\rightarrow} \mathcal{L}_{l} \Gamma .
$$

This can be seen, for example, as follows. Since $\Gamma$ is a finitely generated nilpotent group, there is a central series

$$
\Gamma=\Gamma_{1} \supset \Gamma_{2} \supset \cdots \supset \Gamma_{n}=T_{1} \supset T_{2} \supset \cdots \supset T_{m}=\{1\}
$$

such that $\Gamma_{i} / \Gamma_{i+1} \cong \mathbb{Z}^{r_{i}}\left(r_{i} \in \mathbb{Z}_{>0}, i=1, \ldots, n-1\right)$, and $T_{1}$ is the maximal torsion subgroup of $\Gamma$ (e.g. [Hir], Th. 2.21). As each quotient $\Gamma / \Gamma_{i}(1 \leq i \leq n)$ is $(l)$-good in the sense of Serre [Se], where $(l)$ denotes the class of finite $l$-groups, the pro- $l$ completion functor maps the $\Gamma_{i} / \Gamma_{i+1} \otimes \mathbb{Z}_{l}(1 \leq i \leq n-1)$ isomorphically onto the corresponding successive subquotients of $\hat{\Gamma}(l)$ (cf. e.g. [N2], (1.2.4)). Then we conclude the local isomorphy of $j_{l}$ from the exactness of the Malcev completion functor and of the Lie functor of the $l$-adic analytic groups (cf. [BK], [DSMS]).

A noteworthy remark here is that any automorphism of $\hat{\Gamma}(l)$ induces one of $\mathcal{L}_{l} \Gamma$, hence one of $\mathcal{U}_{l} \Gamma$. 


\section{Weight filtration of surface braid groups.}

(2.4) Now let us introduce the configuration space of a Riemann surface. Let $F_{g}$ be a compact Riemann surface of genus $g$, and let $a_{1}, \ldots, a_{n}$ be $n$ distinct points on $F_{g}(2-2 g-n<0)$. The braid configuration space $F_{g, n}^{(r)}$ of $F_{g, n}:=F_{g}-\left\{a_{1}, \ldots, a_{n}\right\}$ with $r$ strings is defined by

$$
F_{g, n}^{(r)}=\left\{\left(x_{1}, \ldots, x_{r}\right) \in\left(F_{g, n}\right)^{r} \mid x_{i} \neq x_{j}(i \neq j)\right\} .
$$

(2.4.1) If $f_{k}(k=1, \ldots, r)$ denotes the projection morphism $F_{g, n}^{(r)} \rightarrow F_{g, n}^{(r-1)}$ obtained by forgetting the $k$-th component, then it gives a fibration over $F_{g, n}^{(r-1)}$. We shall denote, for simplicity, the discrete fundamental group of $F_{g, n}^{(r)}$ by $\pi_{g, n}^{(r)}$ (when $r=1$ simply by $\pi_{g, n}$ ) and the kernel of the homomorphism $p_{k}=\pi_{1}\left(f_{k}\right)$ by $\phi_{k}$ or $\phi_{k}^{(g, n, r)}$, which is called the $k$-th fibre subgroup of $\pi_{g, n}^{(r)}$. (When speaking about fundamental groups, we often omit references to base points as long as no confusion occurs.) We have then the following exact sequence:

$$
1 \longrightarrow \phi_{k} \longrightarrow \pi_{g, n}^{(r)} \stackrel{p_{k}}{\longrightarrow} \pi_{g, n}^{(r-1)} \longrightarrow 1 \text {. }
$$

As $\phi_{k}$ is isomorphic to the fundamental group of $F_{g}$ minus $(n+r-1)$ points, it is possible to present it as

$$
\phi_{k} \cong\left\langle\begin{array}{c|c}
x_{1}^{(k)}, \cdots, x_{g}^{(k)}, y_{1}^{(k)}, \cdots, y_{g}^{(k)} & {\left[x_{1}^{(k)}, y_{1}^{(k)}\right] \cdots\left[x_{g}^{(k)}, y_{g}^{(k)}\right] z_{1}^{(k)} \cdots z_{n+r}^{(k)}} \\
z_{1}^{(k)}, \cdots, z_{n+r}^{(k)} & =z_{n+k}^{(k)}=1
\end{array}\right\rangle,
$$

where each $z_{j}^{(k)}$ generates an inertia subgroup of a puncture of the fibre of $f_{k}$. As in [NTU], $\S 2$, we shall take these generators so that their parabolic elements satisfy $z_{n+j}^{(k)}=z_{n+k}^{(j)}$ for $1 \leq j, k \leq r$.

(2.4.2) Throughout the present paper, we let $\varepsilon$ represent an integer such that

$$
\varepsilon:= \begin{cases}3 & \text { if }(g, n)=(0,3), \\ 1 & \text { if }(g, n)=(1,1), \\ 0 & \text { otherwise. }\end{cases}
$$

When $\varepsilon \neq 0$, it is useful to introduce auxiliary projections $p_{k}: \pi_{g, n}^{(r)} \rightarrow \pi_{g, n}^{(r-1)}$ and fibre subgroups $\phi_{k}=\operatorname{ker}\left(p_{k}\right)$ for $r<k \leq r+\varepsilon$. In these exceptional cases, we sometimes (re)normalize the presentation of $\phi_{k}$ as

$$
\phi_{k}=\left\langle\begin{array}{c|c}
\tilde{x}_{1}^{(k)}, \cdots, \tilde{x}_{g}^{(k)}, \tilde{y}_{1}^{(k)}, \cdots, \tilde{y}_{g}^{(k)} \mid\left[\tilde{x}_{1}^{(k)}, \tilde{y}_{1}^{(k)}\right] & \cdots\left[\tilde{x}_{g}^{(k)}, \tilde{y}_{g}^{(k)}\right] \tilde{z}_{1}^{(k)} \cdots \tilde{z}_{n+r}^{(k)} \\
\tilde{z}_{1}^{(k)}, \cdots, \tilde{z}_{n+r}^{(k)} & =\tilde{z}_{n-\varepsilon+k}^{(k)}=1
\end{array}\right\rangle
$$

for $1 \leq k \leq r+\varepsilon$ in the following ways. When $(g, n)=(0,3)$, the space $F_{0,3}^{(r)}$ is isomorphic to the moduli space $M_{0, r+3}$ of the $(r+3)$-pointed projective lines. Then, in the notations of [N2], $\S 3, \pi_{0,3}^{(r)}$ is isomorphic to $\Gamma_{0}^{r+3}=\pi_{1}\left(M_{0, r+3}\right)$, which has $r+3$ forgetful projections $p_{1}, \ldots, p_{r+3}$ to $\Gamma_{0}^{r+2}$. We shall regard $p_{1}, \ldots, p_{r}$ of these as the ones introduced in (2.4.1). In terms of the standard generators $\left\{A_{i j}\right\}$ of $\Gamma_{0}^{r+3}$ in loc. cit., the normalized generators are given by $\tilde{z}_{j}^{(k)}=A_{j k}$ for $j=1, \ldots, n+r$. When $(g, n)=(1,1)$, the space $F_{1,1}^{(r)}$ can be written as $\left\{E^{r+1}-\Delta\right\} / E$, where $E$ is an elliptic curve, $\Delta$ is the weak diagonals and $/ E$ means the quotient by the diagonal translations by $E$. Therefore we have $r+1$ forgetful maps $p_{k}: \pi_{1,1}^{(r)} \rightarrow \pi_{1,1}^{(r-1)}$ 
$(1 \leq k \leq r+1)$ obtained by forgetting one of the coordinates of $E^{r+1}-\Delta$ and have the fibre subgroup $\phi_{k}=\operatorname{ker}\left(p_{k}\right)(1 \leq k \leq r+1)$. Here we content ourselves simply by taking normalized generator systems $\left\{\left(\tilde{x}_{1}^{(k)}, \tilde{y}_{1}^{(k)}, \tilde{z}_{j}^{(k)}\right)\right\}$ of $\phi_{k}$ 's to be $\left\{\left(x_{1}^{(k)}, y_{1}^{(k)}, z_{2}^{(k)}, \ldots, z_{r+1}^{(k)}, z_{1}^{(k)}\right)\right\}$ for $1 \leq k \leq r$ and a system satisfying the conjugacy relations $\tilde{z}_{j}^{(r+1)} \sim z_{1}^{(j)}(1 \leq j \leq r)$ for $k=r+1$ (cf. also (2.9)).

(2.5) Recalling that each $\phi_{k} \cong \pi_{g, n+r-1}$ has a good weight filtration of normal subgroups $\left\{\phi_{k}(m)\right\}_{m=1}^{\infty}$ introduced by Oda and Kaneko (cf. [K], [O], [AN], [NT]), we shall introduce the weight filtration $\pi_{g, n}^{(r)}=\pi_{g, n}^{(r)}(1) \supset \pi_{g, n}^{(r)}(2) \supset \cdots$ by setting

$$
\begin{aligned}
\pi_{g, n}^{(r)}(1) & =\pi_{g, n}^{(r)}, \\
\pi_{g, n}^{(r)}(2) & =\left[\pi_{g, n}^{(r)}, \pi_{g, n}^{(r)}\right]\left\langle\phi_{k}(2) \mid k=1, \cdots, r\right\rangle, \\
\pi_{g, n}^{(r)}(m) & =\left\langle\left[\pi_{g, n}^{(r)}\left(m^{\prime}\right), \pi_{g, n}^{(r)}\left(m^{\prime \prime}\right)\right] \mid m^{\prime}+m^{\prime \prime}=m\right\rangle \quad(m \geq 3) .
\end{aligned}
$$

Here $\langle S\rangle$ denotes the minimum closed subgroup containing $S$, and $\left[H_{1}, H_{2}\right]$ denotes the closure of the commutator subgroup of $H_{1}$ and $H_{2}$. This weight filtration of surface braid groups was first studied in [NTU] in the pro- $l$ context, and similar arguments to loc. cit. $\S 2$ imply that the homomorphism $p_{k}: \pi_{g, n}^{(r)} \rightarrow \pi_{g, n}^{(r-1)}(1 \leq$ $k \leq r+\varepsilon)$ induces the exact sequence of graded quotient modules with respect to the weight filtrations:

$$
0 \longrightarrow \mathrm{gr}^{m} \phi_{k} \longrightarrow \mathrm{gr}^{m} \pi_{g, n}^{(r)} \longrightarrow \operatorname{gr}^{m} \pi_{g, n}^{(r-1)} \longrightarrow 0,
$$

and that

$$
\bigcap_{m=1}^{\infty} \pi_{g, n}^{(r)}(m)=\{1\}
$$

Moreover it follows that each $\mathrm{gr}^{m} \pi_{g, n}^{(r)}$ is generated by the $\operatorname{gr}^{m} \phi_{k}(k=1, \ldots, r)$.

(2.6) Since the weight filtration is central, the direct sum

$$
\operatorname{Gr} \pi_{g, n}^{(r)}:=\bigoplus_{m=1}^{\infty} \operatorname{gr}^{m} \pi_{g, n}^{(r)}
$$

has a natural structure of a graded Lie algebra over $\mathbb{Z}$ such that $\left[\mathrm{gr}^{m}, \mathrm{gr}^{\mathrm{m}^{\prime}}\right] \subset$ $\mathrm{gr}^{m+m^{\prime}}$. It is generated by the ideals $\operatorname{Gr} \phi_{k}:=\bigoplus_{m=1}^{\infty} \operatorname{gr}^{m} \phi_{k}(k=1, \ldots, r)$, each of which has a natural presentation induced from that of $\phi_{k}$ (2.4.1) with generators $X_{i}^{(k)}, Y_{i}^{(k)} \in \operatorname{gr}^{1} \Phi_{k}(i=1, \ldots, g), Z_{j}^{(k)} \in \operatorname{gr}^{2} \Phi_{k}(j=1, \ldots, n+r)$ and relations $\sum_{i=1}^{g}\left[X_{i}^{(k)}, Y_{i}^{(k)}\right]+\sum_{j=1}^{n+r} Z_{j}^{(k)}=Z_{n+k}^{(k)}=0$. We shall denote by $\operatorname{Gr}\left(p_{k}\right)$ the Lie homomorphism induced by $p_{k}$. By (2.5.1), it induces an exact sequence of graded Lie algebras:

$$
0 \longrightarrow \operatorname{Gr} \phi_{k} \longrightarrow \operatorname{Gr} \pi_{g, n}^{(r)} \stackrel{\operatorname{Gr}\left(p_{k}\right)}{\longrightarrow} \operatorname{Gr} \pi_{g, n}^{(r-1)} \longrightarrow 0 .
$$

From this, by induction on $r$, we see that $\operatorname{Gr} \pi_{g, n}^{(r)}$ is torsion-free and has trivial center. See Sect. 3 for a more detailed presentation of the Lie algebra structure of $\operatorname{Gr} \pi_{g, n}^{(r)}$. 


\section{Malcev and pro- $l$ completions of surface braid groups.}

(2.7) For each $m \geq 1$, the quotient group $\pi_{g, n}^{(r)} / \pi_{g, n}^{(r)}(m)$ is a torsion free nilpotent group. The Malcev completion and the Malcev Lie algebra of it form projective systems with respect to $m$. We define

$$
\begin{aligned}
& \mathcal{U}_{l}\left(\pi_{g, n}^{(r)}\right):=\varliminf_{m} \mathcal{U}_{l}\left(\pi_{g, n}^{(r)} / \pi_{g, n}^{(r)}(m)\right), \\
& \mathcal{L}_{l}\left(\pi_{g, n}^{(r)}\right):=\varliminf_{m} \mathcal{L}_{l}\left(\pi_{g, n}^{(r)} / \pi_{g, n}^{(r)}(m)\right) .
\end{aligned}
$$

Introduce their weight filtration by taking the weight $-m$ component $\mathcal{U}_{l}\left(\pi_{g, n}^{(r)}\right)(m)$ $\left(\right.$ resp. $\left.\mathcal{L}_{l}\left(\pi_{g, n}^{(r)}\right)(m)\right)(m \geq 1)$ to be the kernel of the canonical mapping to

$$
\mathcal{U}_{l}\left(\pi_{g, n}^{(r)} / \pi_{g, n}^{(r)}(m)\right) \quad\left(\operatorname{resp} . \mathcal{L}_{l}\left(\pi_{g, n}^{(r)} / \pi_{g, n}^{(r)}(m)\right)\right) .
$$

From the fact that the functor of taking Malcev completion is exact, it follows that

$$
\operatorname{gr}^{m} \mathcal{U}_{l}\left(\pi_{g, n}^{(r)}\right) \cong \operatorname{gr}^{m} \mathcal{L}_{l}\left(\pi_{g, n}^{(r)}\right) \cong \operatorname{gr}^{m} \pi_{g, n}^{(r)} \otimes \mathbb{Q}_{l}
$$

We shall denote the pro-l completion of $\pi_{g, n}^{(r)}$ by $\Pi_{g, n}^{(r)}$, and freely use notations similar to [NTU], $\S 2$. For example, the closure of $\phi_{k}(k=1, \ldots, r+\varepsilon)$ in $\Pi_{g, n}^{(r)}$ will be denoted by $\Phi_{k}$. The pro- $l$ braid groups $\Pi_{g, n}^{(r)}$ are equipped with natural weight filtrations $\left\{\Pi_{g, n}^{(r)}(m)\right\}_{m=1}^{\infty}$ (e.g. loc. cit., $\left.\S 2\right)$ satisfying

$$
0 \longrightarrow \operatorname{Gr} \Phi_{k} \longrightarrow \operatorname{Gr} \prod_{g, n}^{(r)} \stackrel{\operatorname{Gr}\left(p_{k}\right)}{\longrightarrow} \operatorname{Gr} \Pi_{g, n}^{(r-1)} \longrightarrow 0 .
$$

Combining (2.6.1), (2.7.2) and a comparison result on weight graduations in the 1-dimensional case due to Asada [A], we obtain

$$
\Pi_{g, n}^{(r)}(m) \cap \pi_{g, n}^{(r)}=\pi_{g, n}^{(r)}(m), \quad \operatorname{gr}^{m} \Pi_{g, n}^{(r)} \cong \operatorname{gr}^{m} \pi_{g, n}^{(r)} \otimes \mathbb{Z}_{l} .
$$

Since $\Pi_{g, n}^{(r)} / \Pi_{g, n}^{(r)}(m)$ is the pro-l completion of $\pi_{g, n}^{(r)} / \pi_{g, n}^{(r)}(m),(2.3 .1)$ gives a homomorphism

$$
j_{l}(m): \Pi_{g, n}^{(r)} / \Pi_{g, n}^{(r)}(m) \longrightarrow \mathcal{U}_{l}\left(\pi_{g, n}^{(r)} / \pi_{g, n}^{(r)}(m)\right)
$$

for each $m$, and they together induce a natural homomorphism

$$
j_{l}: \Pi_{g, n}^{(r)} \longrightarrow \mathcal{U}_{l}\left(\pi_{g, n}^{(r)}\right) .
$$

By comparing (2.7.1) and (2.7.3), we see that these $j_{l}(m)$ and $j_{l}$ are injective. In particular, we may regard $\Pi_{g, n}^{(r)}$ as a subgroup of $\mathcal{U}_{l}\left(\pi_{g, n}^{(r)}\right)$. Then,

$$
\Pi_{g, n}^{(r)}(m)=\Pi_{g, n}^{(r)} \cap \mathcal{U}_{l}\left(\pi_{g, n}^{(r)}\right)(m) \quad(m \geq 1) .
$$

Moreover, identifying the Malcev completion $\mathcal{U}_{l}\left(\phi_{k}\right)$ with the kernel of the projection $\mathcal{U}_{l}\left(\pi_{g, n}^{(r)}\right) \rightarrow \mathcal{U}_{l}\left(\pi_{g, n}^{(r-1)}\right)$ induced from $p_{k}$, we have

$$
\Phi_{k}=\Pi_{g, n}^{(r)} \cap \mathcal{U}_{l}\left(\phi_{k}\right) .
$$

(2.8) Let $\gamma_{m} \pi_{g, n}^{(r)}$ denote the $m$-th lower central subgroup of $\pi_{g, n}^{(r)}(m \geq 1$, and we set $\left.\pi_{g, n}^{(r)}=\gamma_{1} \pi_{g, n}^{(r)}\right)$. Then, by the definitions, our two filtrations are cofinal in such a way that

$$
\pi_{g, n}^{(r)}(2 m) \subset \gamma_{m} \pi_{g, n}^{(r)} \subset \pi_{g, n}^{(r)}(m) \quad(m \geq 1) .
$$


Therefore, $\mathcal{U}_{l}\left(\pi_{g, n}^{(r)}\right)$ and $\mathcal{L}_{l}\left(\pi_{g, n}^{(r)}\right)$ of $(2.7)$ can be written also as

$$
\begin{aligned}
& \mathcal{U}_{l}\left(\pi_{g, n}^{(r)}\right)=\varliminf_{m} \mathcal{U}_{l}\left(\pi_{g, n}^{(r)} / \gamma_{m} \pi_{g, n}^{(r)}\right), \\
& \mathcal{L}_{l}\left(\pi_{g, n}^{(r)}\right)=\varliminf_{m} \mathcal{L}_{l}\left(\pi_{g, n}^{(r)} / \gamma_{m} \pi_{g, n}^{(r)}\right) .
\end{aligned}
$$

On the other hand, if we denote the lower central filtration of the pro- $l$ group $\Pi_{g, n}^{(r)}$ by $\left\{\gamma_{m} \Pi_{g, n}^{(r)}\right\}_{m}$, then the pro-l completion of $\pi_{g, n}^{(r)} / \gamma_{m} \pi_{g, n}^{(r)}$ is isomorphic to $\Pi_{g, n}^{(r)} / \gamma_{m} \Pi_{g, n}^{(r)}$.

Now each automorphism $f$ of $\Pi_{g, n}^{(r)}$ induces an automorphism of $\Pi_{g, n}^{(r)} / \gamma_{m} \Pi_{g, n}^{(r)}$, hence one of $\mathcal{U}_{l}\left(\pi_{g, n}^{(r)} / \gamma_{m} \pi_{g, n}^{(r)}\right)$ via $(2.3 .2)$, denoted $\hat{f}_{m}$. As these $\hat{f}_{m}(m \geq 1)$ are compatible with obvious projections, we obtain a natural mapping

$$
\text { Aut } \Pi_{g, n}^{(r)} \longrightarrow \operatorname{Aut} \mathcal{U}_{l}\left(\pi_{g, n}^{(r)}\right) \cong \operatorname{Aut} \mathcal{L}_{l}\left(\pi_{g, n}^{(r)}\right) .
$$

\section{Classes of (outer) automorphisms of pro- $l$ braid groups.}

(2.9) A full (stereo) braid-like automorphism of $\Pi_{g, n}^{(r)}$ is, by definition, an automorphism of $\Pi_{g, n}^{(r)}$ which permutes the $\Phi_{k}(k=1, \ldots, r+\varepsilon)$ and the conjugacy classes of the cyclic subgroups $\left\langle\tilde{z}_{j}^{(k)}\right\rangle(1 \leq k \leq r+\varepsilon, 1 \leq j \leq n+r)$ among them. This definition coincides with the one given in [NTU] when $\varepsilon=0$. As in loc. cit., (2.9), with each full braid-like automorphism $\sigma \in$ Aut $\Pi_{g, n}^{(r)}$, we may associate two permutations $\pi=\pi_{\sigma} \in S_{n-\varepsilon}, \rho=\rho_{\sigma} \in S_{r+\varepsilon}$ such that

$$
\begin{gathered}
\sigma\left(\Phi_{k}\right)=\Phi_{\rho(k)} \quad(1 \leq k \leq r+\varepsilon), \\
\sigma\left(\left\langle\tilde{z}_{j}^{(k)}\right\rangle\right) \text { is conjugate to }\left\langle\tilde{z}_{\pi(j)}^{(\rho(k))}\right\rangle \quad(1 \leq j \leq n-\varepsilon), \\
\sigma\left(\left\langle\tilde{z}_{n-\varepsilon+i}^{(k)}\right\rangle\right) \text { is conjugate to }\left\langle\tilde{z}_{n-\varepsilon+\rho(i)}^{(\rho(k))}\right\rangle \quad(1 \leq i \leq r+\varepsilon) .
\end{gathered}
$$

The group of all the full (resp. pure, i.e., $\pi_{\sigma}=\rho_{\sigma}=1$ ) braid-like automorphisms of $\Pi_{g, n}^{(r)}$ will be denoted by $\tilde{\Gamma}_{g,[n]}^{[r]+\varepsilon}\left(\right.$ resp. $\left.\tilde{\Gamma}_{g, n}^{(r)+\varepsilon}\right)$, and the subgroups of $\tilde{\Gamma}_{g,[n]}^{[r]+\varepsilon}$ consisting of $\sigma$ with $\rho_{\sigma}=1$ will be denoted by $\tilde{\Gamma}_{g,[n]}^{(r)+\varepsilon}$.

(2.10) We need some more subgroups of Aut $\Pi_{g, n}^{(r)}$ containing $\tilde{\Gamma}_{g,[n]}^{[r]+\varepsilon}$. The first one is $\tilde{\Gamma}_{g,\{n\}}^{\{r\}}$, which consists of the automorphisms $\sigma$ of $\Pi_{g, n}^{(r)}$ preserving each $\Pi_{g, n}^{(r)}(m)$ $(m \geq 1)$ of the weight filtration. The second one is $\tilde{\Gamma}_{g,\{n\}}^{[r]+\varepsilon}$, consisting of all $\sigma \in$ $\tilde{\Gamma}_{g,\{n\}}^{\{r\}}$ such that $\sigma\left(\Phi_{k}\right)=\Phi_{\rho(k)}(k=1, \ldots, r+\varepsilon)$ for some $\rho=\rho_{\sigma} \in S_{r+\varepsilon}$. In summary, we have

$$
\tilde{\Gamma}_{g, n}^{(r)+\varepsilon} \subset \tilde{\Gamma}_{g,[n]}^{(r)+\varepsilon} \subset \tilde{\Gamma}_{g,[n]}^{[r]+\varepsilon} \subset \tilde{\Gamma}_{g,\{n\}}^{[r]+\varepsilon} \subset \tilde{\Gamma}_{g,\{n\}}^{\{r\}} \subset \operatorname{Aut} \Pi_{g, n}^{(r)} .
$$

Also, define $\tilde{\Gamma}_{g,\{n\}}^{(r)+\varepsilon}$ to be $\left\{\sigma \in \tilde{\Gamma}_{g,\{n\}}^{[r]+\varepsilon} \mid \rho_{\sigma}=1\right\}$.

(2.11) For a subgroup $\tilde{\Gamma}$ of $\tilde{\Gamma}_{g,\{n\}}^{\{r\}}$, we introduce its weight filtration by

$$
\tilde{\Gamma}(m)=\left\{\begin{array}{l|l}
\sigma \in \tilde{\Gamma} & \begin{array}{c}
\sigma(x) x^{-1} \in \Pi_{g, n}^{(r)}(1+m)\left(x \in \Pi_{g, n}^{(r)}\right), \\
\sigma\left(x^{\prime}\right) x^{\prime-1} \in \Pi_{g, n}^{(r)}(2+m)\left(x^{\prime} \in \Pi_{g, n}^{(r)}(2)\right)
\end{array}
\end{array}\right\} \quad(m \geq 1) .
$$

When $\tilde{\Gamma}$ contains the inner automorphism group $\operatorname{Int} \Pi_{g, n}^{(r)}$, define the associated outer automorphism group to be $\Gamma:=\tilde{\Gamma} / \operatorname{Int} \Pi_{g, n}^{(r)}$. The following groups corresponding to 
the classes of automorphisms in (2.9)-(2.10) will be considered as practical examples of $\Gamma$ :

$$
\Gamma_{g, n}^{(r)+\varepsilon} \subset \Gamma_{g,[n]}^{(r)+\varepsilon} \subset \Gamma_{g,[n]}^{[r]+\varepsilon}, \Gamma_{g,\{n\}}^{(r)+\varepsilon} \subset \Gamma_{g,\{n\}}^{[r]+\varepsilon} \subset \Gamma_{g,\{n\}}^{\{r\}} \subset \text { Out } \Pi_{g, n}^{(r)}
$$

We shall define the weight filtration of such a $\Gamma$ by projection images: $\Gamma(m)=$ $\tilde{\Gamma}(m) \operatorname{Int} \Pi_{g, n}^{(r)} / \operatorname{Int} \Pi_{g, n}^{(r)}$. As $\operatorname{Gr} \Pi_{g, n}^{(r)}$ is center-free by (2.6.1), (2.7.3), $\Gamma(m)$ turns out to be isomorphic to $\tilde{\Gamma}(m) / \operatorname{Int} \Pi_{g, n}^{(r)}(m)$, where $\operatorname{Int} \Pi_{g, n}^{(r)}(m)$ is the group of the inner automorphisms of $\Pi_{g, n}^{(r)}$ induced by the elements of $\Pi_{g, n}^{(r)}(m)$.

Since each automorphism $\sigma \in \tilde{\Gamma}\left(\subset \tilde{\Gamma}_{g,\{n\}}^{\{r\}}\right)$ preserves the weight filtration of $\Pi_{g, n}^{(r)}$, it induces a graded automorphism $\varpi(\sigma)$ of the graded Lie algebra $\operatorname{Gr} \Pi_{g, n}^{(r)}$. Obviously, the mapping $\varpi: \tilde{\Gamma} \rightarrow$ Aut $\operatorname{Gr} \Pi_{g, n}^{(r)}$ factors through $\Gamma$, and the following exact sequence holds:

$$
1 \longrightarrow \Gamma(1) \longrightarrow \Gamma \stackrel{\varpi}{\longrightarrow} \operatorname{Aut} \operatorname{Gr} \Pi_{g, n}^{(r)} .
$$

Our Theorem D in $\S 3$ will insure that the above map $\varpi$ is surjective, provided that $\tilde{\Gamma}$ contains $\tilde{\Gamma}_{g,[n]}^{[r]+\varepsilon}$.

(2.12) Let us write $\operatorname{Der}^{m}\left(\operatorname{Gr} \Pi_{g, n}^{(r)}\right)$ for the module of the graded derivations of $\operatorname{Gr} \Pi_{g, n}^{(r)}$ of degree $m$, and put

$$
\operatorname{Der}\left(\operatorname{Gr} \Pi_{g, n}^{(r)}\right)=\bigoplus_{m=1}^{\infty} \operatorname{Der}^{m}\left(\operatorname{Gr} \Pi_{g, n}^{(r)}\right)
$$

Let $\tilde{\Gamma}$ be a subgroup of $\tilde{\Gamma}_{g,\{n\}}^{\{r\}}$. Then, each element $\bar{\sigma} \in \operatorname{gr}^{m} \tilde{\Gamma}:=\tilde{\Gamma}(m) / \tilde{\Gamma}(m+1)$ induces a derivation $D_{\bar{\sigma}}$ of degree $m$ by

$$
D_{\bar{\sigma}}\left(x \bmod \Pi_{g, n}^{(r)}(i+1)\right)=\sigma(x) x^{-1} \bmod \Pi_{g, n}^{(r)}(i+m+1) \quad(i \geq 1),
$$

where $\sigma \in \tilde{\Gamma}(m)$ is a representative of $\bar{\sigma}$, and $x \in \Pi_{g, n}^{(r)}(i)$. There is a natural graded Lie structure on

$$
\operatorname{Gr} \tilde{\Gamma}=\bigoplus_{m=1}^{\infty} \operatorname{gr}^{m} \tilde{\Gamma}
$$

induced from the commutator bracket in $\operatorname{Aut} \Pi_{g, n}^{(r)}$, and it is easy to see that the map

$$
\begin{aligned}
D: \operatorname{Gr} \tilde{\Gamma} & \rightarrow \operatorname{Der}\left(\operatorname{Gr} \Pi_{g, n}^{(r)}\right), \\
\bar{\sigma} & \mapsto D_{\bar{\sigma}},
\end{aligned}
$$

is an injective Lie algebra homomorphism. If $\tilde{\Gamma} \supset \operatorname{Int} \Pi_{g, n}^{(r)}$, then $\operatorname{gr}^{m} \Gamma$ is isomorphic to $\operatorname{gr}^{m} \tilde{\Gamma} / \operatorname{gr}^{m} \Pi_{g, n}^{(r)}(2.11)$. So we have also

$$
D^{\prime}: \operatorname{Gr} \Gamma \hookrightarrow \operatorname{Der}\left(\operatorname{Gr} \Pi_{g, n}^{(r)}\right) / \operatorname{Int}\left(\operatorname{Gr} \Pi_{g, n}^{(r)}\right),
$$

where Int denotes the set of inner derivations.

(2.13) There exists an obvious embedding

$$
\operatorname{Der}^{m}\left(\operatorname{Gr} \Pi_{g, n}^{(r)}\right) \hookrightarrow \bigoplus_{i=1}^{\infty} \operatorname{Hom}\left(\operatorname{gr}^{i} \Pi_{g, n}^{(r)}, \operatorname{gr}^{i+m} \Pi_{g, n}^{(r)}\right),
$$


which is equivariant under the action of Aut $\operatorname{Gr} \Pi_{g, n}^{(r)}$ by

$$
\begin{aligned}
& a \cdot D:=a \circ D \circ a^{-1}\left(a \in \operatorname{Aut} \operatorname{Gr} \Pi_{g, n}^{(r)}, D \in \operatorname{Der}^{m} \operatorname{Gr} \Pi_{g, n}^{(r)}\right), \\
& a \cdot f:=a \circ f \circ a^{-1} \quad\left(a \in \operatorname{Aut} \operatorname{Gr} \Pi_{g, n}^{(r)}, f \in \operatorname{Hom}\left(\operatorname{gr}^{i} \Pi_{g, n}^{(r)}, \operatorname{gr}^{i+m} \Pi_{g, n}^{(r)}\right)\right) .
\end{aligned}
$$

Since $\operatorname{Gr} \Pi_{g, n}^{(r)}$ is generated by $\operatorname{gr}^{1} \Pi_{g, n}^{(r)}$ and $\operatorname{gr}^{2} \Pi_{g, n}^{(r)}$, the above embedding holds true even after substituting $\bigoplus_{i=1}^{2}$ for $\bigoplus_{i=1}^{\infty}$. To detect the images of various derivation modules of (2.12) in this "coordinate module" seems an interesting open problem.

Each $\sigma \in \tilde{\Gamma} \subset \tilde{\Gamma}_{g,\{n\}}^{\{r\}}$ induces an automorphism of $\operatorname{gr}^{m} \tilde{\Gamma}$ by conjugation. Since the weight filtration of $\tilde{\Gamma}(1)$ is central, this operation makes $\operatorname{Im}(\varpi)$ of $(2.11)$ act naturally on $\operatorname{gr}^{m} \tilde{\Gamma}$ (and also on $\operatorname{gr}^{m} \Gamma$ when $\operatorname{Int} \Pi_{g, n}^{(r)} \subset \tilde{\Gamma}$.) By an argument similar to $[\mathrm{NT}]$, p.124, we see that (2.12.1) and (2.12.2) are equivariant under the actions of $\operatorname{Im}(\varpi)$.

The reason that filtrations introduced in (2.5), (2.11) are called weight filtrations would be explained as follows. As will be seen in the next section, the matrix group $\operatorname{GSp}\left(2 g, \mathbb{Z}_{l}\right)$ is naturally embedded in Aut $\operatorname{Gr} \Pi_{g, n}^{(r)}$. When $\Pi_{g, n}^{(r)}$ occurs as the pro- $l$ fundamental group of an algebraic configuration space of a curve over $k$, the Galois group $G_{k}$ is mapped into $\operatorname{GSp}\left(2 g, \mathbb{Z}_{l}\right)$ via the $l$-adic Galois representation on the Tate module of a Jacobian variety. Through this map, Gr $\Pi_{g, n}^{(r)}$ and $\operatorname{gr}^{m} \Gamma, \operatorname{gr}^{m} \tilde{\Gamma}$ (for $\tilde{\Gamma} \supset \tilde{\Gamma}_{g, n}^{(r)+\varepsilon}$ ) become $l$-adic Galois modules of pure weight $-m$ in the sense of Grothendieck-Deligne.

\section{Graded automorphisms}

The purpose of this section is to establish the following Theorem $\mathrm{D}$, which plays a crucial role for the proof of Theorem A (4.5).

Let $K$ be a field containing $\mathbb{Q}$, and let $\operatorname{Gr}_{K} \pi_{g, n}^{(r)}=\operatorname{Gr} \pi_{g, n}^{(r)} \otimes_{\mathbb{Z}} K$. We denote by $\operatorname{Aut}\left(\mathrm{Gr}_{K} \pi_{g, n}^{(r)}\right)$ the group of all the graded automorphisms of the graded Lie algebra $\mathrm{Gr}_{K} \pi_{g, n}^{(r)}$ over $K$.

(3.1) Theorem D. Suppose $2-2 g-n<0$ and $r \geq 2$. Then

$$
\operatorname{Aut}\left(\mathrm{Gr}_{K} \pi_{g, n}^{(r)}\right) \cong \operatorname{GSp}(2 g, K) \times S_{n-\varepsilon} \times S_{r+\varepsilon},
$$

where

$$
\varepsilon:=\left\{\begin{array}{l}
3 \quad \text { if }(g, n)=(0,3) \\
1 \quad \text { if }(g, n)=(1,1) \\
0 \quad \text { otherwise }
\end{array}\right.
$$

and $\operatorname{GSp}(0, K):=K^{\times}, S_{0}:=\{1\}$.

This theorem is an expansion of [N2], Lemma (4.1.2) (inspired by P. Deligne), which states that $\operatorname{Aut}\left(\operatorname{Gr}_{K} \pi_{0,3}^{(r)}\right) \cong K^{\times} \times S_{r+3}(r \geq 2)$. We shall prove (3.1) case by case in a way similar to (but more intricate than) loc. cit.

(3.2) We begin by recalling the explicit presentation of $\operatorname{Gr} \pi_{g, n}^{(r)}$ which appeared in [NTU] (cf. [Sc]). Let $X_{i}^{(k)}\left(\right.$ resp. $\left.X_{g+i}^{(k)}, Z_{j}^{(k)}\right)$ denote $x_{i}^{(k)} \bmod \pi_{g, n}^{(r)}(2)\left(\right.$ resp. $y_{i}^{(k)}$ $\left.\bmod \pi_{g, n}^{(r)}(2), z_{j}^{(k)} \bmod \pi_{g, n}^{(r)}(3)\right)(1 \leq k \leq r, 1 \leq i \leq g, 1 \leq j \leq n+r)$. The graded 
Lie algebra $\operatorname{Gr} \pi_{g, n}^{(r)}$ has the following presentation:

generators: $X_{i}^{(k)}, Z_{j}^{(k)} \quad(1 \leq i \leq 2 g, 1 \leq j \leq n+r, 1 \leq k \leq r)$,

relations:

$$
\begin{aligned}
& \sum_{i=1}^{g}\left[X_{i}^{(k)}, X_{i+g}^{(k)}\right]+\sum_{j=1}^{n+r} Z_{j}^{(k)}=0 \quad(1 \leq k \leq r), \\
& Z_{n+k}^{(k)}=0 \quad(1 \leq k \leq r), \quad Z_{n+k^{\prime}}^{(k)}=Z_{n+k}^{\left(k^{\prime}\right)} \quad\left(1 \leq k, k^{\prime} \leq r\right), \\
& {\left[Z_{j}^{(k)}, Z_{j^{\prime}}^{\left(k^{\prime}\right)}\right]=0 \quad\left(\{k+n, j\} \cap\left\{k^{\prime}+n, j^{\prime}\right\}=\emptyset\right),} \\
& {\left[Z_{j}^{(k)}, X_{i}^{\left(k^{\prime}\right)}\right]=0 \quad\left(k \neq k^{\prime} \text { and } j \neq n+k^{\prime}\right),} \\
& {\left[X_{i}^{(k)}, X_{i^{\prime}}^{\left(k^{\prime}\right)}\right]= \begin{cases}0 & \left(k \neq k^{\prime}, i \leq i^{\prime}, i^{\prime} \neq i+g\right), \\
Z_{n+k}^{\left(k^{\prime}\right)} & \left(k \neq k^{\prime}, i^{\prime}=i+g\right) .\end{cases} }
\end{aligned}
$$

By combining (A1)-(A5), we also have

$$
\begin{gathered}
{\left[Z_{j}^{(k)}, Z_{j}^{(h)}\right]=-\left[Z_{j}^{(k)}, Z_{n+h}^{(k)}\right] \quad(1 \leq j \leq n+r, 1 \leq k, h \leq r),} \\
{\left[X_{i}^{(k)}, Z_{n+h}^{(k)}\right]=-\left[X_{i}^{(h)}, Z_{n+k}^{(h)}\right] \quad(1 \leq i \leq 2 g, 1 \leq k, h \leq r) .}
\end{gathered}
$$

Let $\operatorname{Gr}_{K} \phi_{k}:=\operatorname{Gr} \phi_{k} \otimes K$ for each $1 \leq k \leq r$. Then, $\operatorname{Gr}_{K} \phi_{k}$ is an ideal of $\operatorname{Gr}_{K} \pi_{g, n}^{(r)}$ as well as a free Lie subalgebra of it generated by $\left\{X_{i}^{(k)}, Z_{j}^{(k)} ; 1 \leq i \leq 2 g, 1 \leq j \leq\right.$ $n+r\}$. It is also easy to see that

$$
\begin{gathered}
\operatorname{gr}_{K}^{1} \phi_{k}=\bigoplus_{i=1}^{2 g}\left\langle X_{i}^{(k)}\right\rangle \quad(1 \leq k \leq r), \quad \operatorname{gr}_{K}^{1} \pi_{g, n}^{(r)}=\bigoplus_{k=1}^{r} \operatorname{gr}_{K}^{1} \phi_{k} \\
\operatorname{gr}_{K}^{2} \phi_{k} \cap \operatorname{gr}_{K}^{2} \phi_{h}=\left\langle Z_{n+h}^{(k)}\right\rangle \quad(k \neq h) .
\end{gathered}
$$

(Here $\langle S\rangle$ denotes the $K$-linear subspace generated by $S$.) Let $\operatorname{Aut}^{b}\left(\operatorname{Gr}_{K} \pi_{g, n}^{(r)}\right)$ be the group of all the graded automorphisms of $\mathrm{Gr}_{K} \pi_{g, n}^{(r)}$ which permute the $\mathrm{Gr}_{K} \phi_{k}$ $(k=1, \ldots, r)$ and $\left\langle Z_{j}^{(k)}\right\rangle(1 \leq k \leq r, 1 \leq j \leq n+r)$ among them respectively. Then observing the action of $\operatorname{Aut}^{\mathrm{b}}\left(\mathrm{Gr}_{K} \pi_{g, n}^{(r)}\right)$ on the relations (A1-7), we obtain

$$
\operatorname{Aut}^{b}\left(\operatorname{Gr}_{K} \pi_{g, n}^{(r)}\right) \stackrel{\sim}{\longrightarrow} \operatorname{GSp}(2 g, K) \times S_{n} \times S_{r} .
$$

See [NTU], (2.9.2).

By virtue of (3.2.3), in non-exceptional cases, we are reduced to showing that any graded Lie automorphism of $\operatorname{Gr}_{K} \pi_{g, n}^{(r)}$ permutes $\operatorname{Gr}_{K} \phi_{k}(1 \leq k \leq r)$ and $\left\langle Z_{j}^{(k)}\right\rangle$ $(1 \leq k \leq r, 1 \leq j \leq n+r)$. Roughly speaking, in the case of $n=0$, the lines $\left\langle Z_{j}^{(k)}\right\rangle$ are characterized by the $\mathrm{Gr}_{K} \phi_{k}$ 's which are characterized by Lemma (3.3) below, while in the other cases of $n>0$, the $\operatorname{Gr}_{K} \phi_{k}$ 's are characterized by the lines $\left\langle Z_{j}^{(k)}\right\rangle$ which are characterized by Lemmas (3.4-6).

When $Z \in \operatorname{Gr}_{K} \pi_{g, n}^{(r)}$, we let $C(Z)$ denote the centralizer of $Z$ in $\operatorname{Gr}_{K} \pi_{g, n}^{(r)}$, $C^{m}(Z):=C(Z) \cap \operatorname{gr}_{K}^{m} \pi_{g, n}^{(r)}$, and denote by $\mathfrak{Z}=\mathfrak{Z}_{g, n}^{(r)}$ the Lie subalgebra of $\mathrm{Gr}_{K} \pi_{g, n}^{(r)}$ generated by $\left\{Z_{j}^{(k)} ; 1 \leq k \leq r, 1 \leq j \leq n\right\}$. 
Lemma (3.3). Suppose $g \geq 2, n=0, r \geq 2$. Let $X$ be a non-zero element of $\operatorname{gr}_{K}^{1} \pi_{g, 0}^{(r)}$. If $X \in \bigcup_{k=1}^{r} \operatorname{gr}_{K}^{1} \phi_{k}$, then $\operatorname{dim}_{K} C^{1}(X)=(2 g-1)(r-1)+1$. If $X \notin$ $\bigcup_{k=1}^{r} \operatorname{gr}_{K}^{1} \phi_{k}$, then $\operatorname{dim}_{K} C^{1}(X)<(2 g-1)(r-1)+1$.

Lemma (3.4). Suppose $g \geq 1, n \geq 1, r \geq 2$. Let $Z$ be a non-zero element of $\operatorname{gr}_{K}^{2} \pi_{g, n}^{(r)}$. Then the following conditions on $Z$ are equivalent:

(a) $Z \in \mathfrak{Z} \cap \operatorname{gr}_{K}^{2} \phi_{k}$ for some $1 \leq k \leq r$ or $Z \in\left\langle Z_{n+h}^{(k)}\right\rangle$ for some $1 \leq k, h \leq r$,

(b) $\operatorname{dim}_{K} C^{1}(Z) \geq 2 g(r-1)$.

Lemma (3.5). Suppose $g \geq 1, n \geq 2, r \geq 2$. Let $Z$ be a non-zero element of $\mathfrak{Z} \cap \operatorname{gr}_{K}^{2} \phi_{k}$ for some $1 \leq k \leq r$. Then the following conditions on $Z$ are equivalent:

(a) $Z \in\left\langle Z_{j}^{(k)}\right\rangle$ for some $1 \leq j \leq n$,

(b) $\operatorname{dim}_{K}\left(C^{2}(Z) \cap \mathfrak{Z}\right) \geq(n-1)(r-1)+1$.

Lemma (3.6). Suppose $g=0, n \geq 4, r \geq 2$. Let $Z$ be a non-zero element of $\operatorname{gr}_{K}^{2} \pi_{0, n}^{(r)}$. Then the following conditions on $Z$ are equivalent:

(a) $Z \in\left\langle Z_{j}^{(k)}\right\rangle$ for some $1 \leq k \leq r, 1 \leq j \leq n+r$,

(b) $\operatorname{dim}_{K} C^{2}(Z) \geq(n-1)(r-1)+1+\left(\begin{array}{c}r-1 \\ 2\end{array}\right)$.

Proof of Theorem (3.1) by using (3.3-6). We shall separate (3.1) into the following five cases, and prove the theorem case by case:

(1) $g \geq 2, n=0$, (2) $g \geq 1, n \geq 2$, (3) $g \geq 2, n=1$, (4) $g=n=1$, (5) $g=0, n \geq 4$.

For the case $g=0, n=3$, see [N2], $\S$. Let $f$ be any element of Aut $\operatorname{Gr}_{K} \pi_{g, n}^{(r)}$.

(1): $g \geq 2, n=0$. By virtue of Lemma (3.3), $f$ permutes $\operatorname{gr}_{K}^{1} \phi_{k}$. From this together with (A5), (3.2.2), we see that $f \in \operatorname{Aut}^{\mathrm{b}}\left(\mathrm{Gr}_{K} \pi_{g, 0}^{(r)}\right)$. Then (3.2.3) concludes our assertion.

(2): $g \geq 1, n \geq 2$. By Lemma (3.4), $f$ preserves the union of the $\mathfrak{Z} \cap \operatorname{gr}_{K}^{2} \phi_{k}$ $(1 \leq k \leq r)$ and the $\left\langle Z_{n+h}^{(k)}\right\rangle(1 \leq k, h \leq r)$. Since $n \geq 2$, the dimensions of $\mathfrak{Z} \cap \operatorname{gr}_{K}^{2} \phi_{k}$ and $\left\langle Z_{n+h}^{(k)}\right\rangle$ are different. Therefore, $f$ induces a permutation on the $\mathfrak{Z} \cap \operatorname{gr}_{K}^{2} \phi_{k}(1 \leq k \leq r)$ and one on the $\left\langle Z_{n+h}^{(k)}\right\rangle(1 \leq k, h \leq r)$. In particular, $f$ preserves $\mathfrak{Z}$. So we can apply Lemma (3.5) to see that $f$ permutes the flags $\left\langle Z_{j}^{(k)}\right\rangle \subset \mathfrak{Z} \cap \operatorname{gr}_{K}^{2} \phi_{k}(1 \leq k \leq r, 1 \leq j \leq n)$. On the other hand, by (A4), we have

$$
\operatorname{gr}_{K}^{1} \phi_{k}=\bigcap_{\substack{1 \leq h \leq r, h \neq k \\ 1 \leq j \leq n}} C^{1}\left(Z_{j}^{(h)}\right) \text {. }
$$

Thus, $f$ permutes $\operatorname{gr}_{K}^{1} \phi_{k}(1 \leq k \leq r)$. Taking (A5) into consideration, we see that $f$ permutes $\operatorname{Gr}_{K} \phi_{k}(1 \leq k \leq r)$ and obtain the conclusion by (3.2.3).

Before going to the cases $(3),(4),(5)$, we shall prepare the following lemma.

Lemma (3.7). For each $1 \leq k \leq r+1$, let

$$
\mathfrak{z}^{(k)}= \begin{cases}\bigcup_{1 \leq j \leq n+r}\left\langle Z_{j}^{(k)}\right\rangle \subset \operatorname{gr}_{K}^{2} \phi_{k} & (1 \leq k \leq r), \\ \bigcup_{1 \leq h \leq r}\left\langle Z_{1}^{(h)}\right\rangle \subset \operatorname{gr}_{K}^{2} \pi_{g, n}^{(r)} & (k=r+1) .\end{cases}
$$

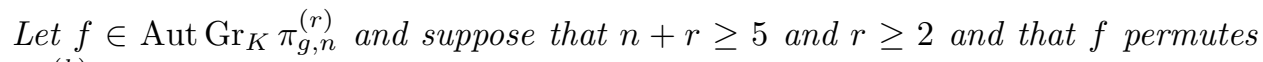
$\left\langle Z_{j}^{(k)}\right\rangle(1 \leq k \leq r, 1 \leq j \leq n+r)$. If $n=0$ or $n \geq 2$, then $f$ permutes $\mathfrak{z}^{(k)}$ $(1 \leq k \leq r)$, and if $n=1$, then $f$ permutes $\mathfrak{z}^{(k)}(1 \leq k \leq r+1)$. 
Proof of Lemma (3.7). To each non-zero $Z_{j}^{(k)}$ we associate a pair of integers $S=$ $\{n+k, j\}$ and write $Z_{S}=Z_{j}^{(k)}$. Then the lines generated by the $Z_{j}^{(k)}$ correspond bijectively to the elements of

$$
\mathfrak{S}=\{S \subset\{1, \ldots, n+r\} \mid \# S=2, S \cap\{n+1, \ldots, n+r\} \neq \emptyset\} .
$$

Then by (A3), (A6) and the freeness of $\operatorname{Gr}_{K} \phi_{k}(1 \leq k \leq r)$, it follows that $\left[Z_{S}, Z_{S^{\prime}}\right]=0$ if and only if $S \cap S^{\prime}=\emptyset$ for $S, S^{\prime} \in \mathfrak{S}$. Therefore, if $F$ denotes the permutation on $\mathfrak{S}$ induced naturally from the action of $f$ on the $\left\langle Z_{j}^{(k)}\right\rangle$, then

$$
\{i, j\} \cap\left\{i^{\prime}, j^{\prime}\right\} \neq \emptyset \Longleftrightarrow F(\{i, j\}) \cap F\left(\left\{i^{\prime}, j^{\prime}\right\}\right) \neq \emptyset .
$$

If we use the assumption $n+r-1 \geq 4$, then we further obtain

$$
\bigcap_{\substack{1 \leq j \leq n+r \\ j \neq n+k}} F(\{n+k, j\}) \neq \emptyset .
$$

The statement for the case $n=0$ follows merely from this observation. If $n \geq 2$, then $n+r-1>r$, so, for any $1 \leq k \leq r$, the common element of (3.7.2) must be $\geq n+1$; hence the $Z$ 's corresponding to the $F(\{n+k, j\})(j \neq n+k, 1 \leq j \leq n+r)$ must be contained in one of $\operatorname{gr}_{K}^{2} \phi_{1}, \ldots, \operatorname{gr}_{K}^{2} \phi_{r}$. Thus we get the conclusion. If $n=1$, then the common element of (3.7.2) could be 1 (for some $k$ ). Taking this possibility into consideration in this case, we obtain the above statement.

(3),(4): $g \geq 1, n=1$. Let us introduce new symbols: $X_{i}^{(r+1)}:=-\sum_{k=1}^{r} X_{i}^{(k)}$ $(1 \leq i \leq 2 g)$ and $Z_{j}^{(r+1)}:=Z_{1}^{(j)}(1 \leq j \leq r)$, and let $\mathrm{Gr}_{K} \phi_{r+1}$ denote the Lie-ideal in $\operatorname{Gr}_{K} \pi_{g, 1}^{(r)}$ generated by $\left\{X_{i}^{(r+1)}, Z_{j}^{(r+1)} ; 1 \leq i \leq 2 g, 1 \leq j \leq r\right\}$. By Lemma (3.4) and the fact that $\mathfrak{Z} \cap \operatorname{gr}_{K}^{2} \phi_{k}$ is 1-dimensional for $n=1$, it follows that

$$
f \text { permutes }\left\langle Z_{h}^{(k)}\right\rangle \quad(1 \leq k, h \leq r+1) .
$$

Set $\operatorname{gr}_{K}^{m} \phi_{r+1}=\operatorname{Gr}_{K} \phi_{r+1} \cap \operatorname{gr}_{K}^{m} \pi_{g, 1}^{(r)}$, and $\mathcal{Z}=\left\{Z_{j}^{(k)} \mid 1 \leq k \leq r, 1 \leq j \leq n+r\right\}$. Then, from (A4), (A7) (see also (3.10.1-2)),

$$
\begin{gathered}
\operatorname{gr}_{K}^{1} \phi_{k}=\bigcap_{Z \in \mathcal{Z} \backslash \mathfrak{z}^{(k)}} C^{1}(Z) \quad(1 \leq k \leq r+1), \\
0=\bigcap_{Z \in \mathfrak{z}^{(k)} \backslash_{\mathfrak{z}^{(l)}}} C^{1}(Z) \cap C^{1}\left(Z_{q}^{(l)}\right) \quad\left(\begin{array}{c}
1 \leq k, l, q \leq r+1, \\
q-1 \neq l \neq k
\end{array}\right) .
\end{gathered}
$$

Using (3.8.1-3) and Lemma (3.7), we see that $f$ permutes $\mathfrak{z}^{(k)}(1 \leq k \leq r+1)$. (The case $r=2$ follows from (3.8.1). For the case $r=3$, we need (3.8.1-3). The case $r \geq 4$ follows from (3.8.1), (3.7).) Then by (3.8.1-2), $f$ permutes $\operatorname{gr}_{K}^{1} \phi_{k}(1 \leq$ $k \leq r+1)$, i.e., there exists a permutation $\tau \in S_{r+1}$ with $f\left(\operatorname{gr}_{K}^{1} \phi_{k}\right)=\operatorname{gr}_{K}^{1} \phi_{\tau(k)}$, $f\left(\mathfrak{z}^{(k)}\right)=\mathfrak{z}^{(\tau(k))}$. On the other hand, we have from (A2), (A5) a relation

$$
\sum_{i=1}^{g}\left[X_{i}^{(r+1)}, X_{g+i}^{(r+1)}\right]+\sum_{j=1}^{r} Z_{j}^{(r+1)}=2(g-1) \sum_{Z \in \mathcal{Z} \backslash \mathfrak{z}^{(r+1)}} Z .
$$

(3): Suppose $g \geq 2$. By (3.2.3) and (3.8.1), it suffices to show $\tau(r+1)=r+1$. In fact, if not, then $\tau(r+1)=k$ for some $k \leq r$, and $f$ maps the LHS of (3.8.4) into $\operatorname{Gr}_{K}\left(\phi_{k}\right)$. Meanwhile, the RHS must be mapped to a sum of non-zero scalar 
multiples of the $Z \in \mathcal{Z} \backslash \mathfrak{z}^{(k)}$, which does not lie in $\operatorname{Gr}_{K}\left(\phi_{k}\right)$. This is a contradiction, and hence $\tau(r+1)=r+1$.

(4): When $g=1$, we have an isomorphism

$$
\mathrm{Gr}_{K} \pi_{1,1}^{(r)} \simeq \operatorname{Gr}_{K} \pi_{1,0}^{(r+1)} / V
$$

where $V$ is the subspace in $\operatorname{gr}_{K}^{1} \pi_{1,0}^{(r+1)}$ generated by the ' $\sum_{k=1}^{r+1} X_{i}^{(k)}$, $(1 \leq i \leq 2 g)$. As $V$ is $\operatorname{GL}(2, K) \times S_{r+1}$-invariant, we have a natural map $\mathrm{GL}(2, K) \times S_{r+1} \rightarrow$ Aut $\mathrm{Gr}_{K} \pi_{1,1}^{(r)}$. From this we may assume $\tau(r+1)=r+1$ without loss of generality. Then, by using (3.2.3), we conclude the proof.

(5): $g=0, n \geq 4$. Let $f$ be any element of $\operatorname{Aut}\left(\mathrm{Gr}_{K} \pi_{0, n}^{(r)}\right)$. Then by Lemma (3.6), $f$ permutes $\left\langle Z_{j}^{(k)}\right\rangle(1 \leq k \leq r, 1 \leq j \leq n+r)$, and then by Lemma (3.7), $f$ permutes $\operatorname{gr}_{K}^{2} \phi_{k}=\left\langle\mathfrak{z}^{(k)}\right\rangle(1 \leq k \leq r)$. Hence $f \in \operatorname{Aut}^{\mathrm{b}}\left(\mathrm{Gr}_{K} \pi_{0, n}^{(r)}\right)$. By (3.2.3), we conclude the statement.

Proofs of Lemmas (3.3-6). In the case of Lemma (3.3), we shall prove the first part by a direct computation using (A1-5) and the second part by induction on $r$. In the cases (3.4)-(3.6), we shall prove $(a) \Rightarrow(b)$ by a direct computation and $(b) \Rightarrow(a)$ by induction on $r$. Let $\mathrm{Gr}_{K} p_{\nu}$ denote $\operatorname{Gr} p_{\nu} \otimes K: \operatorname{Gr}_{K} \pi_{g, n}^{(r)} \rightarrow \operatorname{Gr}_{K} \pi_{g, n}^{(r-1)}$.

Proof of Lemma (3.3). ( $g \geq 2, n=0)$. First, we shall compute $\operatorname{dim}_{K} C^{1}\left(X_{i}^{(k)}\right)$ $(1 \leq k \leq r, 1 \leq i \leq 2 g)$. By virtue of (A5), it follows that $C^{1}\left(X_{i}^{(k)}\right)$ contains $\left\{X_{\alpha}^{(\gamma)} ; \gamma \neq k,|\alpha-i| \neq g\right\}$. On the other hand, the $\left[X_{i}^{(k)}, X_{\alpha}^{(k)}\right],\left[X_{i}^{(k)}, X_{i \pm g}^{(\gamma)}\right]= \pm Z_{\gamma}^{(k)}$ $(1 \leq \alpha \leq 2 g, \alpha \neq i, 1 \leq \gamma \leq r, \gamma \neq k)$ are linearly independent over $K$, as part of a basis of $\operatorname{gr}_{K}^{2} \phi_{k}(g \geq 2)$. (Here \pm is taken according as $i \leq g$ or $i>g$.) Hence

$$
C^{1}\left(X_{i}^{(k)}\right)=\left\langle X_{i}^{(k)}\right\rangle \oplus \bigoplus_{\gamma \neq k,|\alpha-i| \neq g}\left\langle X_{\alpha}^{(\gamma)}\right\rangle .
$$

Thus, $\operatorname{dim}_{K} C^{1}\left(X_{i}^{(k)}\right)=(2 g-1)(r-1)+1$. Since GSp $(2 g, K)$ acts on each $\operatorname{gr}_{K}^{1} \phi_{k}-$ $\{0\}$ transitively (cf. [Di], Chap.II, Prop.1.), the first statement of Lemma (3.3) follows.

Next, we shall show that $\operatorname{dim}_{K} C^{1}(X)<(2 g-1)(r-1)+1$ for every $X \in$ $\operatorname{gr}_{K}^{1} \pi_{g, 0}^{(r)}-\bigcup_{k=1}^{r} \operatorname{gr}_{K}^{1} \phi_{k}$.

Step 1: $r=2$. We notice that $\mathrm{Gr}_{K} p_{1}$ induces the following exact sequence:

$$
0 \rightarrow C(X) \cap \operatorname{gr}_{K}^{1} \phi_{1} \rightarrow C^{1}(X) \rightarrow C^{1}\left(\operatorname{Gr}_{K} p_{1}(X)\right)\left(\subset \operatorname{Gr}_{K} \pi_{g, n}^{(r-1)}\right) .
$$

Let $d_{1}=\operatorname{dim}_{K}\left(C(X) \cap \operatorname{gr}_{K}^{1} \phi_{1}\right), d_{2}=\operatorname{dim}_{K}\left(C^{1}\left(\operatorname{Gr}_{K} p_{1}(X)\right)\right)$. Then, it suffices to show that

(i) $d_{1} \leq 2 g-2$, and

(ii) $d_{2}=1$.

We first show (i). Supposing contrarily $d_{1} \geq 2 g-1$, we shall deduce $X \in \operatorname{gr}_{K}^{1} \phi_{2}$ to get a contradiction. Since $\operatorname{dim}_{K} A d(X)\left(\operatorname{gr}_{K}^{1} \phi_{1}\right)=2 g-d_{1} \leq 1,\left[X_{i}^{(1)}, X\right]$ and $\left[X_{i^{\prime}}^{(1)}, X\right]$ are linearly dependent for $i \neq i^{\prime}$. Especially, we have $0=c_{i}\left[X_{i}^{(1)}, X\right]+$ $c_{i+g}\left[X_{i+g}^{(1)}, X\right]$ for some $\left(c_{i}, c_{i+g}\right) \in K^{2}-\{0\}(1 \leq i \leq g)$. Expressing $X$ as 
GALOIS RIGIDITY OF PRO-l PURE BRAID GROUPS OF ALGEBRAIC CURVES 1093

$$
\begin{aligned}
& \sum_{\alpha=1}^{2 g} a_{\alpha} X_{\alpha}^{(1)}+a_{\alpha}^{\prime} X_{\alpha}^{(2)}\left(a_{\alpha}, a_{\alpha}^{\prime} \in K\right), \text { we obtain } \\
& 0=c_{i}\left[X_{i}^{(1)}, X\right]+c_{i+g}\left[X_{i+g}^{(1)}, X\right] \\
& \quad=\sum_{\alpha \neq i} c_{i} a_{\alpha}\left[X_{i}^{(1)}, X_{\alpha}^{(1)}\right]+\sum_{\alpha \neq i+g} c_{i+g} a_{\alpha}\left[X_{i+g}^{(1)}, X_{\alpha}^{(1)}\right]+\left(c_{i} a_{i+g}^{\prime}-c_{i+g} a_{i}^{\prime}\right) Z_{2}^{(1)} .
\end{aligned}
$$

Here, notice that, since $g \geq 2,\left[X_{i}^{(1)}, X_{\alpha}^{(1)}\right],\left[X_{i+g}^{(1)}, X_{\alpha}^{(1)}\right](1 \leq \alpha \leq 2 g, \alpha \neq i, i+g)$, $\left[X_{i}^{(1)}, X_{i+g}^{(1)}\right]$ and $Z_{2}^{(1)}$ are linearly independent over $K$ for fixed $i$, as part of a basis of $\operatorname{gr}_{K}^{2} \phi_{1}$. Hence $c_{i} a_{\alpha}=c_{i+g} a_{\alpha}=0(\alpha \neq i, i+g)$. As $\left(c_{i}, c_{i+g}\right) \neq(0,0)$, we obtain $a_{\alpha}=0(\alpha \neq i, i+g)$. But since this holds for $i=1,2, a_{\alpha}=0$ for all $\alpha$, i.e., $X \in \operatorname{gr}_{K}^{1} \phi_{2}$.

As for (ii), we have only to see that $\operatorname{dim}_{K}\left(C_{\mathrm{Gr}_{K} \pi_{g, 0}}^{1}\left(X^{\prime}\right)\right)=1$ for $0 \neq X^{\prime} \in$ $\operatorname{gr}_{K}^{1} \pi_{g, 0}$. But since $\operatorname{GSp}(2 g, K)$ acts on $\operatorname{gr}^{1} \Pi_{g, 0}-\{0\}$ transitively, this reduces to the obvious case $X^{\prime}=X_{1}$.

Step 2: $r \geq 3$. We choose $h(1 \leq h \leq r)$ such that $\operatorname{Gr}_{K} p_{h}(X) \notin \bigcup_{k=1}^{r-1} \operatorname{gr}_{K}^{1} \phi_{k}$. (This is possible because $X \notin \bigcup_{k=1}^{r} \operatorname{gr}_{K}^{1} \phi_{k}$ and $r \geq 3$.) We note that $\operatorname{Gr}_{K} p_{h}$ induces the following exact sequence:

$$
0 \rightarrow C(X) \cap \operatorname{gr}_{K}^{1} \phi_{h} \rightarrow C^{1}(X) \rightarrow C^{1}\left(\operatorname{Gr}_{K} p_{h}(X)\right)\left(\subset \operatorname{Gr}_{K} \pi_{g, n}^{(r-1)}\right) .
$$

By the induction hypothesis, $\operatorname{dim}_{K} C^{1}\left(\operatorname{Gr}_{K} p_{h}(X)\right)<(2 g-1)(r-2)+1$. On the other hand, by virtue of (3.9), we can see that $\bigcap_{i=1}^{2 g} C^{1}\left(X_{i}^{(h)}\right)=0$, which implies $\operatorname{dim}_{K}\left(C(X) \cap \operatorname{gr}_{K}^{1} \phi_{h}\right) \leq 2 g-1$. Thus $\operatorname{dim}_{K} C^{1}(X)<(2 g-1)(r-1)+1$.

Proof of Lemma (3.4). $(g \geq 1, n \geq 1) \cdot(\mathbf{a}) \Rightarrow(\mathbf{b})$ : It suffices only to observe from (A4) and (A7) that for any $Z_{n+k^{\prime}}^{(k)}\left(k<k^{\prime}\right)$ and any $Z \in \mathfrak{Z} \cap \operatorname{gr}_{K}^{2} \phi_{k}-\{0\}$,

$$
\begin{aligned}
& C^{1}\left(Z_{n+k^{\prime}}^{(k)}\right)=\bigoplus_{i=1}^{2 g}\left(\left\langle X_{i}^{(k)}+X_{i}^{\left(k^{\prime}\right)}\right\rangle \oplus \bigoplus_{h \neq k, k^{\prime}}\left\langle X_{i}^{(h)}\right\rangle\right), \\
& C^{1}(Z)=\left\langle X_{i}^{(h)} ; 1 \leq h \leq r, h \neq k, 1 \leq i \leq 2 g\right\rangle .
\end{aligned}
$$

(b) $\Rightarrow(\mathbf{a})$ : We shall prove this by induction on $r$, by using the exact sequence

$$
0 \rightarrow C(Z) \cap \operatorname{gr}_{K}^{1} \phi_{1} \rightarrow C^{1}(Z) \rightarrow C^{1}\left(\operatorname{Gr}_{K} p_{1}(Z)\right)\left(\subset \operatorname{Gr}_{K} \pi_{g, n}^{(r-1)}\right)
$$

induced from $\mathrm{Gr}_{K} p_{1}$.

Step 1: $r=2$. Suppose $Z \notin\left\langle Z_{n+2}^{(1)}\right\rangle$. Since $\operatorname{gr}_{K}^{2} \phi_{1} \cap \operatorname{gr}_{K}^{2} \phi_{2}=\left\langle Z_{n+2}^{(1)}\right\rangle$, we may assume $\operatorname{Gr}_{K} p_{1}(Z) \neq 0$ by symmetry. By the assumption (b), $\operatorname{dim}_{K} C^{1}(Z) \geq 2 g$. Since $\operatorname{Gr}_{K} \pi_{g, n}$ is a free Lie algebra, $\operatorname{dim}_{K} C^{1}\left(\operatorname{Gr}_{K} p_{1}(Z)\right)=0$. Hence $C^{1}(Z)=$ $\operatorname{gr}_{K}^{1} \phi_{1}$ for $\operatorname{dim}_{K} \operatorname{gr}_{K}^{1} \phi_{1}=2 g$. Thus $\left[X_{\alpha}^{(1)}, Z\right]=0(1 \leq \alpha \leq 2 g)$. We shall take

$$
\left\{\left[X_{\alpha}^{(k)}, X_{\alpha^{\prime}}^{(k)}\right], Z_{\beta}^{(k)}, Z_{n+2}^{(1)} \mid 1 \leq \alpha<\alpha^{\prime} \leq 2 g, 1 \leq \beta \leq n-1, k=1,2\right\}
$$

as a basis of $\operatorname{gr}_{K}^{2} \pi_{g, n}^{(2)}$ and

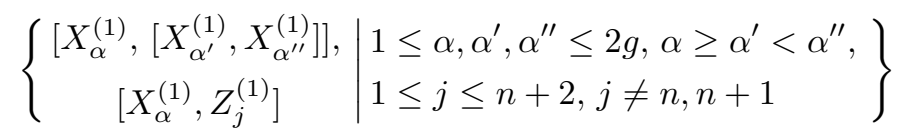


as a basis of $\operatorname{gr}_{K}^{3} \phi_{1}$. Express $Z$ as $Y_{1}+Y_{2}+c Z_{n+2}^{(1)}$ by using the above basis such that $Y_{k}=\sum a_{i, i^{\prime}}^{(k)}\left[X_{i}^{(k)}, X_{i^{\prime}}^{(k)}\right]+\sum b_{j}^{(k)} Z_{j}^{(k)}(k=1,2)$. Then, from (A4), (A5) and (A7) it follows that

$$
\begin{aligned}
& {\left[X_{\alpha}^{(1)}, Y_{2}\right]=} \\
& \left\{\begin{array}{l}
-\sum_{1 \leq i<\alpha+g} a_{i, \alpha+g}^{(2)}\left[X_{i}^{(1)}, Z_{n+2}^{(1)}\right]+\sum_{\alpha+g<i^{\prime} \leq 2 g} a_{\alpha+g, i^{\prime}}^{(2)}\left[X_{i^{\prime}}^{(1)}, Z_{n+2}^{(1)}\right] \quad(1 \leq \alpha \leq g), \\
+\sum_{1 \leq i<\alpha-g} a_{i, \alpha-g}^{(2)}\left[X_{i}^{(1)}, Z_{n+2}^{(1)}\right]-\sum_{\alpha-g<i^{\prime} \leq 2 g} a_{\alpha-g, i^{\prime}}^{(2)}\left[X_{i^{\prime}}^{(1)}, Z_{n+2}^{(1)}\right] \quad(g<\alpha \leq 2 g) .
\end{array}\right.
\end{aligned}
$$

In particular, $\left[X_{\alpha}^{(1)}, Y_{2}\right] \in\left[\operatorname{gr}_{K}^{1} \phi_{1}, Z_{n+2}^{(1)}\right]$; hence $0=\left[X_{\alpha}^{(1)}, Z\right] \equiv\left[X_{\alpha}^{(1)}, Y_{1}\right]$ modulo the ideal $\left(Z_{n+2}^{(1)}\right)$. Since $\operatorname{Gr}_{K} \phi_{1} /\left(Z_{n+2}^{(1)}\right) \cong \mathrm{Gr}_{K} \Pi_{g, n}$ is free, $Y_{1} \equiv 0 \bmod \left(Z_{n+2}^{(1)}\right)$; but then $Y_{1}=0$. Returning to $0=\left[X_{\alpha}^{(1)}, Z\right]=\left[X_{\alpha}^{(1)}, Y_{2}\right]+c\left[X_{\alpha}^{(1)}, Z_{n+2}^{(1)}\right]$, we see that

$$
c= \begin{cases}a_{\alpha, \alpha+g}^{(2)} & (1 \leq \alpha \leq g), \\ a_{\alpha-g, \alpha}^{(2)} & (g+1 \leq \alpha \leq 2 g),\end{cases}
$$

and $a_{i j}^{(2)}=0(i, j \leq g$ or $i, j>g), a_{i, \alpha+g}^{(2)}=a_{\alpha-g, i^{\prime}}^{(2)}=0\left(i, i^{\prime} \neq \alpha\right)$, which amount to all $a_{i j}^{(2)}$ and $c$ being zero. Thus $Z=\sum_{j<n} b_{j}^{(2)} Z_{j}^{(2)} \in \mathfrak{Z} \cap \operatorname{gr}_{K}^{2} \phi_{2}$.

Step 2: $r \geq 3$. As $\operatorname{gr}_{K}^{2} \phi_{1} \cap \operatorname{gr}_{K}^{2} \phi_{2} \cap \operatorname{gr}_{K}^{2} \phi_{3}=0$, we may assume $\operatorname{Gr}_{K} p_{1}(Z) \neq 0$ by symmetry. By assumption

$$
\operatorname{dim}_{K} C^{1}(Z) \geq 2 g(r-1) .
$$

Since $d_{1}:=\operatorname{dim}_{K}\left(C(Z) \cap \operatorname{gr}_{K}^{1} \phi_{1}\right) \leq 2 g$, we have

$$
d_{2}:=\operatorname{dim}_{K} C^{1}\left(\operatorname{Gr}_{K} p_{1}(Z)\right) \geq 2 g(r-2) .
$$

Then, by induction, $d_{2}=2 g(r-2)$, hence $d_{1}=2 g$, i.e., $C(Z) \supset \operatorname{gr}_{K}^{1} \phi_{1}$. The induction hypothesis also enables us to express $Z$ in one of the following forms:

$$
Z \equiv \begin{cases}\sum_{j=1}^{n} \lambda_{j} Z_{j}^{(k)} & \left(2 \leq k \leq r, \lambda_{1}, \ldots, \lambda_{n} \in K\right), \\ \lambda Z_{n+h}^{(k)} & \left(2 \leq k \leq h \leq r, \lambda \in K^{\times}\right),\end{cases}
$$

modulo $\operatorname{gr}_{K}^{2} \phi_{1}$. But since each RHS above centralizes $\operatorname{gr}_{K}^{1} \phi_{1}$ by (A4), the above $\equiv$ must be real $=$ in $\operatorname{gr}_{K}^{2} \pi_{g, n}^{(r)}$. Thus we conclude the proof.

Proof of Lemma (3.5). ( $g \geq 1, n \geq 2)$. (a) $\Rightarrow(\mathbf{b})$ : By (A3), we have for each $1 \leq \beta \leq n$,

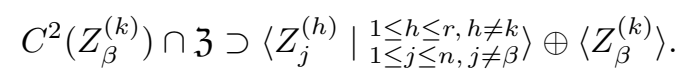

In order to show the converse inclusion, let $Z \in C^{2}\left(Z_{\beta}^{(k)}\right) \cap \mathfrak{Z}$. Then, expressing $Z$ as $\sum_{\substack{1 \leq h \leq r \\ 1 \leq j \leq n}} b_{j}^{(h)} Z_{j}^{(h)}$, we obtain from (A3),(A6)

$$
0=\left[Z, Z_{\beta}^{(k)}\right]=\sum_{\substack{1 \leq j \leq n \\ j \neq \beta}} b_{j}^{(k)}\left[Z_{j}^{(k)}, Z_{\beta}^{(k)}\right]-\sum_{\substack{1 \leq h \leq r \\ h \neq k}} b_{\beta}^{(h)}\left[Z_{n+h}^{(k)}, Z_{\beta}^{(k)}\right] .
$$


By the freeness of $\operatorname{Gr}_{K} \phi_{k}$ and $g \geq 1$, we obtain $b_{j}^{(k)}=b_{\beta}^{(h)}=0(j \neq \beta, h \neq k)$. Hence the desired inclusion holds.

(b) $\Rightarrow$ (a): We may assume that $\operatorname{Gr}_{K} p_{1}(Z) \neq 0$, i.e., $Z \in \mathfrak{Z} \cap \operatorname{gr}^{2} \phi_{k}$ for some $k \geq 2$. We note that $\mathrm{Gr}_{K} p_{1}$ induces the following exact sequence:

$$
0 \rightarrow C_{\mathfrak{Z}}^{2}(Z) \cap \operatorname{gr}_{K}^{2} \phi_{1} \rightarrow C_{\mathfrak{Z}}^{2}(Z) \rightarrow C^{2}\left(\operatorname{Gr}_{K} p_{1}(Z)\right),
$$

where $C_{\mathfrak{Z}}^{m}(*)=C^{m}(*) \cap \mathfrak{Z}$.

Step 1: $r=2$. Writing $Z$ as $\sum_{j=1}^{n} b_{j} Z_{j}^{(2)}$, we shall show that the coefficients vanish except for one. By the assumption (b), $\operatorname{dim}_{K} C_{3}^{2}(Z) \geq n$. Since $\operatorname{Gr}_{K} \pi_{g, n}$ is a free Lie algebra, $\operatorname{dim}_{K} C^{2}\left(\operatorname{Gr}_{K} p_{1}(Z)\right)=1$. Hence

$$
n-1 \leq \operatorname{dim}_{K} C_{\mathfrak{Z}}^{2}(Z) \cap \operatorname{gr}_{K}^{2} \phi_{1} \leq n,
$$

which implies that $\left[Z_{\beta}^{(1)}, Z\right]$ and $\left[Z_{\beta^{\prime}}^{(1)}, Z\right]$ are linearly dependent for any $1 \leq \beta<$ $\beta^{\prime} \leq n$. For such a pair $\left(\beta, \beta^{\prime}\right)$, pick $\left(a_{\beta}, a_{\beta^{\prime}}\right) \in K^{2}-\{0\}$ such that $a_{\beta}\left[Z_{\beta}^{(1)}, Z\right]+$ $a_{\beta^{\prime}}\left[Z_{\beta^{\prime}}^{(1)}, Z\right]=0$. Then, by using (A3) and (A6), we obtain

$$
a_{\beta} b_{\beta}\left[Z_{\beta}^{(1)}, Z_{n+2}^{(1)}\right]+a_{\beta^{\prime}} b_{\beta^{\prime}}\left[Z_{\beta^{\prime}}^{(1)}, Z_{n+2}^{(1)}\right]=0 .
$$

As $\mathrm{Gr}_{K} \phi_{1}$ is free, $a_{\beta} b_{\beta} Z_{\beta}^{(1)}+a_{\beta^{\prime}} b_{\beta^{\prime}} Z_{\beta^{\prime}}^{(1)}=c Z_{n+2}^{(1)}$ for some $c \in K$. As $g \geq 1$, $Z_{\beta}^{(1)}, Z_{\beta^{\prime}}^{(1)}$ and $Z_{n+2}^{(1)}$ are linearly independent over $K$. Hence $a_{\beta} b_{\beta}=a_{\beta^{\prime}} b_{\beta^{\prime}}=c=0$. Thus $b_{\beta}=0$ or $b_{\beta^{\prime}}=0$ for any $1 \leq \beta<\beta^{\prime} \leq n$. This concludes (a).

Step 2: $r \geq 3$. Let $d_{1}=\operatorname{dim}_{K} C_{\mathfrak{Z}}(Z) \cap \operatorname{gr}_{K}^{2} \phi_{1}$ and $d_{2}=\operatorname{dim}_{K} C^{2}\left(\operatorname{Gr}_{K} p_{1}(Z)\right)$.

Case 1: $d_{2} \leq(n-1)(r-2)$. Then by $(\mathrm{b}), d_{1}=n$. Hence $\left[Z_{\beta}^{(1)}, Z\right]=0$ $(1 \leq \beta \leq n)$. Expressing $Z$ as $\sum_{j=1}^{n} b_{j} Z_{j}^{(k)}$ and using (A3) and (A6), we obtain $0=b_{\beta}\left[Z_{\beta}^{(1)}, Z_{n+k}^{(1)}\right]$. Hence $b_{\beta}=0$ for any $1 \leq \beta \leq n$, which is a contradiction.

Case 2: $d_{2}>(n-1)(r-2)$. As $\operatorname{Gr}_{K} p_{1}(Z) \subset \mathfrak{Z}_{g, n}^{(r-1)}$, by the induction hypothesis, it follows that $d_{2}=(n-1)(r-2)+1$. Hence $n-1 \leq d_{1} \leq n$. Then in the same way as in Step 1, we conclude (a).

Proof of Lemma (3.6). $(g=0, n \geq 4)$. (a) $\Rightarrow(\mathbf{b})$ : It is easy to see that $\operatorname{Gr}_{K} \pi_{0, n}^{(r)}=\operatorname{Gr}_{K} \phi_{k}+C\left(Z_{j}^{(k)}\right), \operatorname{Gr}_{K} \phi_{k} \cap C\left(Z_{j}^{(k)}\right)=\left\langle Z_{j}^{(k)}\right\rangle$ for $j \neq n+k$. Hence

$$
\begin{aligned}
\operatorname{dim}_{K} C^{2}\left(Z_{j}^{(k)}\right) & =\operatorname{dim}_{K} \operatorname{gr}_{K}^{2} \pi_{0, n}^{(r)}-\operatorname{dim}_{K} \operatorname{gr}_{K}^{2} \phi_{k}+1 \\
& =(n-1)(r-1)+\left(\begin{array}{c}
r-1 \\
2
\end{array}\right)+1 .
\end{aligned}
$$

(b) $\Rightarrow(\mathbf{a})$ : We again start from the following exact sequence induced from $\mathrm{Gr}_{K} p_{1}$ :

$$
0 \rightarrow C(Z) \cap \operatorname{gr}_{K}^{2} \phi_{1} \rightarrow C^{2}(Z) \rightarrow C^{2}\left(\operatorname{Gr}_{K} p_{1}(Z)\right)\left(\subset \operatorname{Gr}_{K} \pi_{0, n}^{(r-1)}\right) .
$$

We denote $d_{1}=\operatorname{dim}_{K} C(Z) \cap \operatorname{gr}_{K}^{2} \phi_{1}, d_{2}=\operatorname{dim}_{K} C^{2}\left(\operatorname{Gr}_{K} p_{1}(Z)\right)$.

Step 1: $r=2$. We may assume $\operatorname{Gr}_{K} p_{1}(Z) \neq 0$ without loss of generality. Write $Z$ as $\sum_{\beta=1}^{n-1} b_{\beta}^{(1)} Z_{\beta}^{(1)}+\sum_{\beta=1}^{n} b_{\beta}^{(2)} Z_{\beta}^{(2)}$. By the assumption (b), $\operatorname{dim}_{K} C^{2}(Z) \geq n$, while, as $\operatorname{Gr}_{K} \pi_{0, n}$ is free, $d_{2}=1$. Hence $n-1 \leq d_{1} \leq n$, which implies that, for any pair $j, j^{\prime} \neq n+1\left(j \neq j^{\prime}\right),\left[Z_{j}^{(1)}, Z\right]$ and $\left[Z_{j^{\prime}}^{(1)}, Z\right]$ are linearly dependent. Let $1 \leq j<j^{\prime} \leq n$, and pick $\left(a_{j}, a_{j^{\prime}}\right) \neq(0,0)$ such that $a_{j}\left[Z_{j}^{(1)}, Z\right]+a_{j^{\prime}}\left[Z_{j^{\prime}}^{(1)}, Z\right]=0$. 
Then, by using (A3) and (A6), we get

$$
0=\left[a_{j} Z_{j}^{(1)}+a_{j^{\prime}} Z_{j^{\prime}}^{(1)}, \sum_{\beta=1}^{n-1} b_{\beta}^{(1)} Z_{\beta}^{(1)}\right]-a_{j} b_{j}^{(2)}\left[Z_{j}^{(1)}, Z_{n+2}^{(1)}\right]-a_{j^{\prime}} b_{j^{\prime}}^{(2)}\left[Z_{j^{\prime}}^{(1)}, Z_{n+2}^{(1)}\right] .
$$

If $j<j^{\prime} \supsetneqq n$, then, taking $\left\{Z_{\beta}^{(1)} ; 1 \leq \beta \leq n+2, \beta \neq n, n+1\right\}$ as a free basis of $\operatorname{Gr}_{K} \phi_{1}$, we obtain the following relations

$$
\left\{\begin{array}{c}
a_{j} b_{j^{\prime}}^{(1)}=a_{j^{\prime}} b_{j}^{(1)}, \\
a_{j} b_{\beta}^{(1)}=0 \quad\left(1 \leq \beta \leq n-1, \beta \neq j, j^{\prime}\right), \\
a_{j^{\prime}} b_{\beta}^{(1)}=0 \quad\left(1 \leq \beta \leq n-1, \beta \neq j, j^{\prime}\right) .
\end{array}\right.
$$

Then, since $n \geq 4$, we may move $j, j^{\prime}<n$ to obtain $b_{\beta}^{(1)}=0(1 \leq \beta \leq n-1)$. Returning to $(*)$ for general $j, j^{\prime} \leq n$, we then have

$$
a_{j} b_{j}^{(2)}\left[Z_{j}^{(1)}, Z_{n+2}^{(1)}\right]+a_{j^{\prime}} b_{j^{\prime}}^{(2)}\left[Z_{j^{\prime}}^{(1)}, Z_{n+2}^{(1)}\right]=0 .
$$

As $\left[Z_{j}^{(1)}, Z_{n+2}^{(1)}\right]$ and $\left[Z_{j^{\prime}}^{(1)}, Z_{n+2}^{(1)}\right]$ are linearly independent over $K$, we obtain $a_{j} b_{j}^{(2)}=$ $a_{j^{\prime}} b_{j^{\prime}}^{(2)}=0$. Thus $b_{j}^{(2)}$ or $b_{j^{\prime}}^{(2)}=0$ for any $1 \leq j<j^{\prime} \leq n$, which implies (a).

Step 2: $r \geq 3$. We may assume that $\operatorname{Gr}_{K} p_{1}(Z) \neq 0$. As $d_{1} \leq \operatorname{dim}_{K} \operatorname{gr}_{K}^{2} \phi_{1}=$ $n+r-2$, (b) implies $d_{2} \geq(n-1)(r-2)+\left(\begin{array}{c}r-2 \\ 2\end{array}\right)$.

Case 1: $d_{2}>(n-1)(r-2)+\left(\begin{array}{c}r-2 \\ 2\end{array}\right)$. By the induction hypothesis, we may write $Z$ in the form

$$
Z=b Z_{\beta}^{(k)}+Z_{1}
$$

where $2 \leq k \leq r, 1 \leq \beta \leq n+r(\beta \neq n+1, n+k), b \in K^{\times}$and $Z_{1} \in \operatorname{gr}_{K}^{2} \phi_{1}$. We shall prove $Z_{1}=0$. By the induction hypothesis, $d_{2}=(n-1)(r-2)+1+\left(\begin{array}{c}r-2 \\ 2\end{array}\right)$. From this and (b), we obtain $n+r-3 \leq d_{1} \leq n+r-2$, which implies linear dependence of $\left[Z_{j}^{(1)}, Z\right]$ and $\left[Z_{j^{\prime}}^{(1)}, Z\right]$ for every pair of $j, j^{\prime} \neq n+1\left(j \neq j^{\prime}\right)$. Meanwhile, as $n \geq 4$, $r \geq 3$, there exist $j_{1}, j_{2}, j_{3} \in\{1, \ldots, n+r\} \backslash\{n+1, n+k, \beta\}$ such that $Z_{j_{1}}^{(1)}, Z_{j_{2}}^{(1)}$ and $Z_{j_{3}}^{(1)}$ are linearly independent, each of which, by (A3), commutes with $Z_{\beta}^{(k)}$. Thus, we can construct, for each $(p, q) \in\{(1,2),(1,3),(2,3)\}$, a nontrivial linear combination $Y_{p, q}$ of $Z_{j_{p}}^{(1)}$ and $Z_{j_{q}}^{(1)}$ killed by $A d\left(Z_{1}\right)$. Then, since $\operatorname{Gr}_{K} \phi_{1}$ is free, $Z_{1} \in\left\langle Y_{p, q}\right\rangle$ for each $(p, q)$. By the construction, $\bigcap_{(p, q)}\left\langle Y_{p, q}\right\rangle=0$; hence $Z_{1}=0$.

Case 2: $d_{2}=(n-1)(r-2)+\left(\begin{array}{c}r-2 \\ 2\end{array}\right)$. In this case, (b) implies $d_{1}=n+r-2$. Then $\left[Z_{j}^{(1)}, Z\right]=0$ for any $1 \leq j \leq n+r$. Taking $\left\{Z_{\beta}^{(k)} ; 1 \leq k \leq r, 1 \leq \beta \leq n+k-2\right\}$ as a basis of $\operatorname{gr}_{K}^{2} \pi_{0, n}^{(r)}$, and, expressing $Z$ as $\sum_{\substack{1 \leq k \leq k \leq r \\ 1 \leq n+2}} a_{\beta}^{(k)} Z_{\beta}^{(k)}$, we obtain the following $n+r-1$ equations $\left(E_{j}\right)(1 \leq j \leq n+r, j \neq n+1)$ :

$$
\begin{aligned}
0 & =\left[Z_{j}^{(1)}, Z\right]=\sum_{\beta=1}^{n-1} a_{\beta}^{(1)}\left[Z_{j}^{(1)}, Z_{\beta}^{(1)}\right]+\sum_{k=3}^{r} a_{n+1}^{(k)}\left[Z_{j}^{(1)}, Z_{n+k}^{(1)}\right] \\
& - \begin{cases}\sum_{k=2}^{r} a_{j}^{(k)}\left[Z_{j}^{(1)}, Z_{n+k}^{(1)}\right] & (1 \leq j \leq n), \\
\sum_{k=j-n+2}^{r} a_{j}^{(k)}\left[Z_{j}^{(1)}, Z_{n+k}^{(1)}\right]+\sum_{\beta=1}^{j-2} a_{\beta}^{(j-n)}\left[Z_{j}^{(1)}, Z_{\beta}^{(1)}\right] & (n+2 \leq j \leq n+r) .\end{cases}
\end{aligned}
$$


For $2 \leq h \leq r$, consider the claims:

$$
\left(P_{h}\right): \quad a_{\beta}^{(k)}= \begin{cases}0 & (1 \leq k \leq h, 1 \leq \beta \leq n+k-2) \\ a_{n+1}^{(k)} & (h+1 \leq k \leq r, 1 \leq \beta \leq n+h-1) .\end{cases}
$$

By choosing a convenient free basis of $\operatorname{Gr}_{K} \phi_{1}$ for each $j$, we can deduce $\left(P_{2}\right)$ from $\left(E_{1}\right), \ldots,\left(E_{n}\right)$, and $\left(P_{h+1}\right)$ from $\left(P_{h}\right),\left(E_{n+h}\right)$ for each $h$. Thus we obtain $\left(P_{r}\right)$, namely $Z=0$. This is a contradiction, and this case does not occur under our assumption $Z \neq 0$.

\section{Galois Representations}

(4.1) Now we shall study Galois representations. Let $X$ be a projective smooth curve over a number field $k$ of genus $g$, and let $S \subset X$ be a $k$-subset of closed points with geometric cardinality $n$. If an embedding of $\bar{k}$ into $\mathbb{C}$ is fixed, then the complex points $X(\mathbb{C})$ form a compact Riemann surface of genus $g$, on which $S(\mathbb{C})$ lies as a subset of cardinality $n$. We shall regard $X(\mathbb{C}), S(\mathbb{C})$ as $F_{g},\left\{a_{1}, \ldots, a_{n}\right\}$ of $\S 2(2.4)$ respectively. Assume $2-2 g-n<0$ so that $C:=X-S$ becomes a hyperbolic curve, and let $\varepsilon$ be as in (2.4.2). We shall introduce, for $r \geq 1$, the $r$-th configuration space $C^{(r)}$ by

$$
C^{(r)}:=\left\{\left(x_{1}, \ldots, x_{r}\right) \in C^{r} \mid x_{i} \neq x_{j} \text { for } i \neq j\right\},
$$

so that $C^{(r)}(\mathbb{C})=F_{g, n}^{(r)}$. Then, by the well-known comparison theorem $([\mathrm{G} 3])$, we may naturally identify $\pi_{1}\left(C^{(r)} \otimes \bar{k}\right)$ with the profinite completion of $\pi_{g, n}^{(r)}=\pi_{1}\left(F_{g, n}^{(r)}\right)$, whose maximal pro- $l$ quotient is nothing but $\Pi_{g, n}^{(r)}$ of $\S 2$ for a fixed prime $l$.

Since $C^{(r)}$ is defined over $k$, we have a natural (exterior) Galois representation

$$
\varphi_{C}^{(r)}: G_{k} \longrightarrow \text { Out } \Pi_{g, n}^{(r)} .
$$

Meanwhile, each $k$-automorphism of $C^{(r)}$ induces naturally an element of Out $\Pi_{g, n}^{(r)}$ centralizing the Galois image $\varphi_{C}^{(r)}\left(G_{k}\right)$. A basic problem arising here is then to understand closely the diagram

$$
G_{k} \stackrel{\varphi_{C}^{(r)}}{\longrightarrow} \text { Out } \Pi_{g, n}^{(r)} \longleftarrow \operatorname{Aut}_{k} C^{(r)}
$$

where the images of both arrows centralize each other. The purpose of this section is, then, to estimate the Galois centralizer Out $G_{k} \Pi_{g, n}^{(r)}$ (associated with $C_{/ k}^{(r)}$ and $l)$, which is by definition the centralizer of the image of $\varphi_{C}^{(r)}$.

Recall that we have defined subclasses of the outer automorphisms in $\S 2$ as follows:

$$
\Gamma_{g,[n]}^{[r]+\varepsilon} \subset \Gamma_{g,\{n\}}^{[r]+\varepsilon} \subset \Gamma_{g,\{n\}}^{\{r\}} \subset \text { Out } \Pi_{g, n}^{(r)}
$$

We know that the Galois image $\varphi_{C}^{(r)}\left(G_{k}\right)$ is contained in $\Gamma_{g,[n]}^{[r]+\varepsilon}$ for algebro-geometric reasons, while, as will be seen in Lemma (4.3) below, the Galois centralizer is contained in $\Gamma_{g,\{n\}}^{[r]+\varepsilon}$. We first need to show the following lemma.

Lemma (4.2).

$$
\text { Out }_{G_{k}} \Pi_{g, n}^{(r)} \subset \Gamma_{g,\{n\}}^{\{r\}}
$$


Proof. Let $f \in \operatorname{Out}_{G_{k}} \Pi_{g, n}^{(r)}$, and choose a lift $\tilde{f} \in \operatorname{Aut}_{g, n}^{(r)}$ of $f$. It suffices to show that $\tilde{f}$ preserves the weight filtration of $\Pi_{g, n}^{(r)}$. First we consider the naturally induced Galois representation

$$
\bar{\varphi}: G_{k} \longrightarrow \operatorname{Aut}^{b}\left(\operatorname{Gr}_{\mathbb{Q}_{l}} \Pi_{g, n}^{(r)}\right)=\operatorname{GSp}\left(2 g, \mathbb{Q}_{l}\right) \times S_{n} \times S_{r}
$$

and choose $\sigma \in G_{k}$ such that $\bar{\varphi}(\sigma)$ is of the form $\left(a_{\sigma} 1_{2 g}, i d, i d\right)$ for some $a_{\sigma} \in 1+l \mathbb{Z}_{l}$. (This is possible by Bogomolov's theorem [Bog].) Pick a lift $\tilde{\sigma} \in \operatorname{Aut}\left(\Pi_{g, n}^{(r)}\right)$ of $\varphi_{C}^{(r)}(\sigma)$, and regard both $\tilde{f}$ and $\tilde{\sigma}$ as automorphisms of $\mathcal{L}_{l}\left(\pi_{g, n}^{(r)}\right)$ by (2.8.4). Noticing that $\tilde{\sigma}$ acts on the $m$-th graded quotient of $\mathcal{L}_{l}\left(\pi_{g, n}^{(r)}\right)$ by $a_{\sigma}^{m}$-multiplication $(m \geq 1)$, we obtain a 'weight graduation' decomposition with respect to $\tilde{\sigma}$ :

$$
\mathcal{L}_{l}\left(\pi_{g, n}^{(r)}\right)(m)=\prod_{j=m}^{\infty} W_{j}^{(r)} \quad(m \geq 1)
$$

where $W_{j}^{(r)}$ is the pure weight $-j$ component:

$$
W_{j}^{(r)}=\left\{\lambda \in \mathcal{L}_{l}\left(\pi_{g, n}^{(r)}\right) \mid \tilde{\sigma}(\lambda)=a_{\sigma}^{j} \lambda\right\} .
$$

On the other hand, since these $\tilde{f}$ and $\tilde{\sigma}$ commute with each other modulo the inner automorphism group, there exists $u \in \operatorname{Int} \mathcal{L}_{l}\left(\pi_{g, n}^{(r)}\right)$ with

$$
\tilde{f} \tilde{\sigma} u=\tilde{\sigma} \tilde{f} .
$$

Observe here that $\tilde{\sigma}$ and $u$ preserve the weight filtration, and consider their truncations $\tilde{\sigma}_{N}, u_{N} \in \operatorname{Aut} \mathcal{L}_{l}\left(\pi_{g, n}^{(r)} / \pi_{g, n}^{(r)}(N)\right)$ respectively for $N \geq 1$. This observation enables us to employ an argument inspired by P. Deligne (cf. [N2], §4): Namely, by Borel's lemma ([Bor], III, Prop.9.3 (2)), there exists a unique $v_{N} \in$ $\operatorname{Int} \mathcal{L}_{l}\left(\pi_{g, n}^{(r)} / \pi_{g, n}^{(r)}(N)\right)$ with $\tilde{\sigma}_{N} u_{N}=v_{N} \tilde{\sigma}_{N} v_{N}^{-1}$. So we obtain $v=\left(v_{N}\right) \in \operatorname{Int} \mathcal{L}_{l}\left(\pi_{g, n}^{(r)}\right)$ with $\tilde{f} v \tilde{\sigma}=\tilde{\sigma} \tilde{f} v$. From this it follows that $\tilde{f} v$ commutes with $\tilde{\sigma}$. Thus, this $\tilde{f} v$ preserves all components $W_{j}^{(r)}$, and hence the weight filtration of $\mathcal{L}_{l}\left(\pi_{g, n}^{(r)}\right)$. Since $v \in \operatorname{Int} \mathcal{L}_{l}\left(\pi_{g, n}^{(r)}\right)$ preserves the weight filtration, $\tilde{f}$ must preserve that of $\Pi_{g, n}^{(r)}$ by (2.7.4).

Now, we shall show

\section{Lemma (4.3).}

$$
\operatorname{Out}_{G_{k}} \Pi_{g, n}^{(r)} \subset \Gamma_{g,\{n\}}^{[r]+\varepsilon} .
$$

Proof. Let us fix $\sigma \in G_{k}$ as in the proof of Lemma (4.2) and pick a lift $\tilde{\sigma}^{(r)}$ of $\varphi_{C}^{(r)}(\sigma)$ to Aut $\Pi_{g, n}^{(r)}$. For each $1 \leq k \leq r+\varepsilon$, we have an exact sequence

$$
1 \longrightarrow \Phi_{k} \longrightarrow \Pi_{g, n}^{(r)} \stackrel{p_{k}}{\longrightarrow} \Pi_{g, n}^{(r-1)} \longrightarrow 1,
$$

and $\tilde{\sigma}^{(r)}$ preserves $\Phi_{k}$. Thus, it induces an automorphism $\tilde{\sigma}_{k}^{(r-1)}$ on $\Pi_{g, n}^{(r-1)}$. Using these $\tilde{\sigma}^{(r)}$ and $\tilde{\sigma}_{k}^{(r-1)}(1 \leq k \leq r+\varepsilon)$, we obtain associated weight graduation decompositions

$$
\mathcal{L}_{l}\left(\pi_{g, n}^{(r)}\right)=\prod_{m=1}^{\infty} W_{m}^{(r)} \quad \text { and } \quad \mathcal{L}_{l}\left(\pi_{g, n}^{(r-1)}\right)=\prod_{m=1}^{\infty} W_{m, k}^{(r-1)}
$$


such that (the natural extension of) $p_{k}$ maps the pure weight $-m$ component $W_{m}^{(r)}$ onto $W_{m, k}^{(r-1)}$. Denote its kernel by $U_{m, k} \subset W_{m}^{(r)}$. Then, we obtain a commutative diagram of exact sequences:

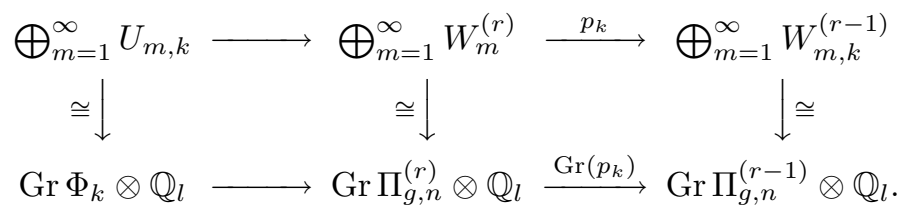

Let $f$ be an arbitrary element of Out $G_{k} \Pi_{g, n}^{(r)}$. Then, as in the proof of Lemma (4.2), we can choose its lift $\tilde{f} \in \operatorname{Aut}\left(\Pi_{g, n}^{(r)}\right)$ and $v \in \operatorname{Int} \mathcal{L}_{l}\left(\pi_{g, n}^{(r)}\right)$ such that $\tilde{f} v$ commutes with $\tilde{\sigma}^{(r)}$. Then $\tilde{f} v$ induces an automorphism of

$$
\bigoplus_{m=1}^{\infty} W_{m}^{(r)} \cong \operatorname{Gr} \Pi_{g, n}^{(r)} \otimes \mathbb{Q}_{l},
$$

hence an element of $\operatorname{GSp}\left(2 g, \mathbb{Q}_{l}\right) \times S_{n-\varepsilon} \times S_{r+\varepsilon}$ by Theorem D (3.1). This means that $\tilde{f} v$ permutes the fibre ideals $\mathcal{L}_{l}\left(\phi_{k}\right)=$ closure of $\bigoplus_{m=1}^{\infty} U_{m, k}(1 \leq k \leq r+\varepsilon)$. Since $v \in \operatorname{Int} \mathcal{L}_{l}\left(\pi_{g, n}^{(r)}\right)$ itself has the same property, we conclude that $\tilde{f}$ permutes $\Phi_{k}=\Pi_{g, n}^{(r)} \cap \mathcal{L}_{l}\left(\phi_{k}\right)(1 \leq k \leq r+\varepsilon)\left(\right.$ cf. (2.7.5)). Hence $f \in \Gamma_{g,\{n\}}^{[r]+\varepsilon}$.

Theorem (4.4). There exists a natural injective homomorphism

$$
\mathrm{Out}_{G_{k}} \Pi_{g, n}^{(r)} \longrightarrow \operatorname{GSp}\left(2 g, \mathbb{Z}_{l}\right) \times S_{n-\varepsilon} \times S_{r+\varepsilon} .
$$

Moreover if $\varepsilon=0,1$ (2.4.2), then it induces an injective homomorphism

$$
\operatorname{Out}_{G_{k}} \Pi_{g, n}^{(r)} \hookrightarrow \operatorname{Out}_{G_{k}} \Pi_{g, n} \times S_{r+\varepsilon} .
$$

Proof. By (4.2), (2.11.1) and Theorem D (3.1), we have a natural homomorphism

$$
\varpi: \operatorname{Out}_{G_{k}} \Pi_{g, n}^{(r)} \longrightarrow \operatorname{Aut}\left(\operatorname{Gr}_{\mathbb{Q}_{l}} \Pi_{g, n}^{(r)}\right)=\operatorname{GSp}\left(2 g, \mathbb{Q}_{l}\right) \times S_{n-\varepsilon} \times S_{r+\varepsilon} .
$$

Since the Galois centralizer is a priori of weight zero (cf. [NT], $\S 3$ ), the map $\varpi$ turns out to be injective by (2.13). Thus the first assertion follows. Next, let $\varepsilon=0,1$, and let $E_{1}$ be $\varpi^{-1}\left(\operatorname{GSp}(2 g) \times S_{n-\varepsilon}\right)$. Then, since $S_{r+\varepsilon}$ is naturally included in our Galois centralizer $\operatorname{Out}_{G_{k}} \Pi_{g, n}^{(r)}$, we have

$$
\text { Out }_{G_{k}} \Pi_{g, n}^{(r)} \cong E_{1} \times S_{r+\varepsilon} \hookrightarrow \operatorname{GSp}\left(2 g, \mathbb{Q}_{l}\right) \times S_{n-\varepsilon} \times S_{r+\varepsilon} .
$$

Now the projection morphism of $C^{(r)}$ to its first component $C$ induces a surjection of $\Pi_{g, n}^{(r)}$ onto $\Pi_{g, n}$, and hence a homomorphism

$$
\psi: \Gamma_{g,\{n\}}^{(r)+\varepsilon} \longrightarrow \text { Out } \Pi_{g, n}
$$

By Lemma (4.3), the group $E_{1}$ is contained in $\Gamma_{g,\{n\}}^{(r)+\varepsilon}$, and is, via the above $\psi$, mapped into Out $_{G_{k}} \Pi_{g, n}$. But we know that this latter Galois centralizer is embedded into $\Gamma_{g,[n]}$ by the 'anabelian' weight argument in $\pi_{1}(C)$, and then into $\operatorname{GSp}(2 g) \times S_{n}$ by the linear weight argument in $\Gamma_{g,[n]}$ (cf. loc. cit., §3, or [N4], $\S 1)$. Then, the image $\psi\left(E_{1}\right) \subset \operatorname{GSp}(2 g) \times S_{n}$ must coincide with $E_{1} \cong \varpi\left(E_{1}\right) \subset$ $\operatorname{GSp}(2 g) \times S_{n-\varepsilon}$ in a canonical way; hence $\psi$ is injective on $E_{1}: \psi\left(E_{1}\right) \cong E_{1}$. From this, we conclude the second assertion. 
(4.5) Theorem A. Let $C$ be a nonsingular hyperbolic curve over a number field $k$ such that $C_{\bar{k}} \not \mathbf{P}^{1}-\{0,1, \infty\}$. Then there exists a sequence of injective homomorphisms

$$
\operatorname{Aut}_{k} C \times S_{r+\varepsilon} \hookrightarrow \operatorname{Aut}_{k} C^{(r)} \hookrightarrow \operatorname{Out}_{G_{k}} \pi_{1}\left(C_{\bar{k}}^{(r)}\right)(l) \hookrightarrow \operatorname{Out}_{G_{k}} \pi_{1}\left(C_{\bar{k}}\right)(l) \times S_{r+\varepsilon},
$$

where $\varepsilon=1$ if $C$ is an elliptic curve minus one $k$-rational point, and 0 otherwise.

Remark (4.6). As in [N2], Th. B, one can consider the $l$-adic Lie version of Grothendieck's conjecture by substituting $\mathcal{L}_{l}\left(\pi_{g, n}^{(r)}\right)$ for $\Pi_{g, n}^{(r)}$. When $n=0,1$, most of our arguments go in exactly similar ways for the Lie version of Theorem A. However, no supporting examples for the 1-dimensional case are known for the Lie version.

Proof of Theorem A (4.5). According to Theorem (4.4), we have only to show that the natural mapping

$$
\Psi: \operatorname{Aut}_{k}\left(C^{(r)}\right) \rightarrow \operatorname{Out}_{G_{k}} \Pi_{g, n}^{(r)}
$$

is an injection. Let us employ an 'intermediate pro-nilpotent technique' with some arguments from [N3]. Put $U=C^{(r)}$ and let $\pi_{1}$ denote the discrete fundamental group $\pi_{g, n}^{(r)}$ of the associated complex manifold with $U$. Moreover we denote the pro-l (resp. pro-nilpotent) completion of $\pi_{1}$ by $\pi_{1}^{\text {pro-l }}$ (resp. $\pi_{1}^{n i l p}$ ). Then $\pi_{1}^{\text {nilp }}=$ $\prod_{l} \pi_{1}^{\text {pro-l }}$ is (universally) center-free. Since $U$ can be embedded into some semiabelian variety, we may apply [N3], Lemma 4.4 and Grothendieck's argument (cf. Proof of Th. 2.1 of loc. cit.) to see that the natural mapping

$$
\mathrm{Aut}_{k} U \longrightarrow \text { Out } \pi_{1}^{n i l p}=\prod_{l \text { prime }} \text { Out } \pi_{1}^{\text {pro-l }}
$$

is an injection. By Lemma (4.2), the image of $\operatorname{Aut}_{k} U$ in each Out $\pi_{1}^{\text {pro-l}}$ is contained in $\Gamma_{g,\{n\}}^{\{r\}}$, and then, by Theorem D (3.1) and a weight argument, there exists an injective mapping

$$
\operatorname{Aut}_{k} U \hookrightarrow \prod_{l \text { prime }}\left(\operatorname{GSp}\left(2 g, \mathbb{Q}_{l}\right) \times S_{n-\varepsilon} \times S_{r+\varepsilon}\right) .
$$

On the other hand, any automorphism in $\operatorname{Aut}_{k} U$ induces an outer automorphism of $\pi_{g, n}^{(r)}=\pi_{1}(U(\mathbb{C})$ ), which preserves the weight filtration by Lemma (4.2) and (2.7.3). Therefore the above injection should factor through the discrete level mapping

$$
\operatorname{Aut}_{k} U \longrightarrow \operatorname{Aut}\left(\operatorname{Gr}_{\mathbb{Q}} \pi_{g, n}^{(r)}\right) \cong \operatorname{GSp}(2 g, \mathbb{Q}) \times S_{n-\varepsilon} \times S_{r+\varepsilon},
$$

which insures that

$$
\operatorname{Aut}_{k} U \longrightarrow \operatorname{GSp}\left(2 g, \mathbb{Z}_{l}\right) \times S_{n-\varepsilon} \times S_{r+\varepsilon}
$$

is already injective. Thus, $\operatorname{Aut}_{k} U \hookrightarrow$ Out $\pi_{1}^{\text {pro-l }}$ for every prime $l$.

\section{REFERENCES}

[A] M.Asada, On the filtration of topological and pro-l mapping class groups of punctured Riemann surfaces, J. Math. Soc. Japan 48 (1996), 13-36. CMP 96:04

[AN] M.Asada, H.Nakamura, On graded quotient modules of mapping class groups of surfaces, Israel J. Math. 90 (1995), 93-113. MR 96j:57015

[Bog] F.A.Bogomolov, Points of finite order on an abelian variety, Math. USSR Izvestiya 17 (1981), 55-72. MR 81m:14031

[Bor] A.Borel, Linear Algebraic Groups, Second enlarged edition, (1st edition from Benjamin 1969), Springer, 1991. MR 92d:20001 
[BK] A.K.Bousfield, D.M.Kan, Homotopy limits, completions and localizations, Lect. Notes in Math., vol. 304, Springer, 1972. MR 51:1825

[De] P.Deligne, Le groupe fondamental de la droite projective moins trois points, The Galois Group over $Q$, ed. by Y.Ihara, K.Ribet, J.-P.Serre, Springer, 1989, pp. 79-297. MR 90m:14016

[Di] J.Dieudonné, Sur les groupes classiques, Actual. Scient. et Ind., n ${ }^{\circ}$ 1040, Hermann, Paris, 1948. MR 9:494c

[DSMS] J.D.Dixon, M.P.F.du Sautoy, A.Mann, D.Segal, Analytic pro-p groups, London Math. Soc. Lect. Notes Series, vol. 157, Cambridge Univ. Press, 1991. MR 94e:20037

[G1] A.Grothendieck, Letter to G.Faltings, 1983.

[G2] A.Grothendieck, Esquisse d'un Programme, mimeographed note (1984).

[G3] A.Grothendieck, Revêtement Etales et Groupe Fondamental (SGA1), Lecture Notes in Math. 224 (1971). MR 50:7129

[Hir] K.A.Hirsch, On infinite soluble groups II, Proc. London Math. Soc. (2) 44 (1933), 336344.

[Ih] Y.Ihara, Automorphisms of pure sphere braid groups and Galois representations, The Grothendieck Festschrift, Volume II, Birkhäuser, 1990, pp. 353-373. MR 92k:20077

[IhK] Y.Ihara, M.Kaneko, Pro-l pure braid groups of Riemann surfaces and Galois representations, Osaka J. Math. 29 (1992), 1-19. MR 93i:14022

[Iv] N.V.Ivanov, Algebraic properties of mapping class groups of surfaces, Geometric and Algebraic Geometry., Banach Center Publ., 1986, pp. 15-35. MR 89a:57009

[K] M.Kaneko, Certain automorphism groups of pro-l fundamental groups of punctured Riemann surfaces., J. Fac. Sci. Univ. Tokyo 36 (1989), 363-372. MR 90j:14019

[KT] Y.Kawahara, T.Terasoma, Galois-Torelli theorems for hyperplane arrangements, in preparation.

[Ma] M.Matsumoto, On Galois representations on profinite braid groups of curves, J. reine angew. Math. 474 (1996), 169-219. CMP 96:13

[M1] S.Mochizuki, The profinite Grothendieck conjecture for closed hyperbolic curves over number fields, J. Math. Sci., Univ. Tokyo 3 (1996), 571-627. CMP 97:07

[M2] S.Mochizuki, The local pro-p Grothendieck conjecture for hyperbolic curves, Preprint RIMS-1045 (1995), RIMS-1097 (1996).

[N1] H.Nakamura, On Galois automorphisms of the fundamental group of the projective line minus three points, Math. Z. 206 (1991), 617-622. MR 92i:14014

[N2] Galois rigidity of pure sphere braid groups and profinite calculus, J. Math. Sci., Univ. Tokyo 1 (1994), 71-136. MR 96e:14021

[N3] , Galois rigidity of algebraic mappings into some hyperbolic varieties, Internat. J. Math. 4 (1993), 421-438. MR 94f:14011

[N4] On exterior Galois representations associated with open elliptic curves, J. Math. Sci., Univ. Tokyo 2 (1995), 197-231. MR 97a:11086

[N5]_, Coupling of universal monodromy representations of Galois- Teichmüller modular groups, Math. Ann. 304 (1996), 99-119. MR 97a:14026

[NT] _ H.Tsunogai, Some finiteness theorems on Galois centralizers in pro-l mapping class groups, J. reine angew Math. 441 (1993), 115-144. MR 94g:14005

[NTU] _ N.Takao and R.Ueno, Some stability properties of Teichmüller modular function fields with pro-l weight structures, Math. Ann. 302 (1995), 197-213. MR 96h:14041

[O] T.Oda, Galois actions on the nilpotent completion of the fundamental group of an algebraic curve, Advances in Number Theory, F.Q.Gouvêa, N.Yui eds., Clarendon Press, Oxford, 1993, pp. 213-232. CMP 96:06

[P] F.Pop, On Grothendieck's conjecture of birational anabelian geometry, Ann. of Math. 138 (1994), 145-182; Part 2, Ann. of Math. (to appear). MR 94m:14007

[Q] D.Quillen, Rational homotopy Theory, Ann. of Math. 90 (1969), 205-295. MR 41:2678

[Sc] G.P.Scott, Braid groups and the group of homeomorphisms of a surface, Proc. Camb. Phil. Soc. 68 (1970), 605-617. MR 42:3786

[Se] J.P.Serre, Cohomologie Galoisienne, 4th ed., Lecture Notes in Math. 5 (1973). MR 53:8030

[Tk] N.Takao, Braid monodromies on proper curves and Galois representations, in preparation. 
[Tm] A.Tamagawa, The Grothendieck conjecture for affine curves, Compositio Math. (to appear).

[W] R.B.Warfield, Jr, Nilpotent Groups, Lect. Notes in Math., vol. 513, Springer, 1976. MR 53:13413

Department of Mathematical Sciences, University of Tokyo, Komaba, Meguro-ku, TOKYO 153, JAPAN

E-mail address: h-naka@ms.u-tokyo.ac.jp

Research Institute for Mathematical Sciences, Kyoto University, Kitashirakawa, КYОTO 606-01, JAPAN

E-mail address: takao@kurims.kyoto-u.ac.jp 